\title{
Aerodynamic Shape Optimization of an Adaptive Morphing Trailing Edge Wing
}

\author{
Zhoujie Lyu* \\ Joaquim R. R. A. Martins ${ }^{\dagger}$ \\ Department of Aerospace Engineering, University of Michigan, Ann Arbor, MI
}

\begin{abstract}
Adaptive morphing trailing edge wings have the potential to reduce the fuel burn of transport aircraft. In this paper, we quantify the aerodynamic performance benefits of a morphing trailing using aerodynamic design optimization. The aerodynamic model solves the Reynolds-averaged NavierStokes equations with a Spalart-Allmaras turbulence model. A gradient-based optimization algorithm is used in conjunction with an adjoint method that computes the required derivatives. The baseline geometry is optimized using a multipoint formulation with 192 shape design variables. The drag coefficient is minimized subject to lift, pitching moment, geometric constraints, and a $2.5 \mathrm{~g}$ maneuver bending moment constraint. The trailing edge of the wing is optimized based on the multipoint optimized wing. The trailing edge morphing is parameterized using 90 design variables that are optimized independently for each flight condition. A total of 407 trailing edge optimizations are performed with different flight conditions to span the entire flight envelope. We observed $1 \%$ drag reduction at on-design conditions, and $5 \%$ drag reduction near off-design conditions. The effectiveness of the trailing edge morphing is demonstrated by comparing with the optimized results of a hypothetical fully morphing wing. In addition, we compute the fuel burn reductions for a number of flights using the optimization results. A 1\% cruise fuel burn reduction is achieved using adaptive morphing trailing edge for a typical long-haul twin-aisle mission.
\end{abstract}

\section{Introduction}

With increasing fuel prices, airlines are seeking more efficient aircraft. Research in aircraft design is therefore placing an increasing emphasis on fuel-burn reduction. One of the fuel-burn reduction strategies that is currently used on modern jetliners, such as the Boeing 787, is the use of cruise flaps: A small amount of trailing edge flap and aileron droop is used to optimize the aerodynamic performance at different cruise conditions. The trailing edge devices can alter the spanloads over the course of a flight to reduce drag; however, cruise flaps have limited degrees-of-freedom that may impact the optimal performance. Morphing trailing edge devices, such as those developed by FlexSys, could address this issue. The FlexSys FlexFoil allows independent changes of camber and flap angles at each spanwise location using a smooth morphing surface with no gaps [1,2]. The morphing trailing edge (TE) has a high technology readiness level and could be retrofited onto existing aircraft to reduce the drag as much as possible for each flight condition.

Previous studies on morphing trailing edges have focused on the design of the morphing mechanism, actuators, and structure $[1,2,3]$. In previous aerodynamic studies of the morphing trailing edge, only low-fidelity methods were used $[4,5]$. However, small geometry changes, such as the cruise flap extension, require high-fidelity simulations to fully quantify the tradeoff between induced drag and other sources of drag. In this paper, we use a high-fidelity aerodynamic model based on the Reynolds-averaged Navier-Stokes (RANS) equations to examine this tradeoff. The boundary layer is well-resolved and a Spalart-Allmaras turbulence model is used.

We performed a multipoint aerodynamic shape optimization of the wing to provide an optimized baseline for the trailing edge optimization. Determining the optimal trailing edge shape at each spanwise location for each flight condition is a challenging design task. We use a gradient-based numerical optimization algorithm together with an efficient adjoint implementation [6] to optimize the morphing for the different flight conditions. A database of optimal

*PhD Candidate, Department of Aerospace Engineering, University of Michigan, AIAA Student Member

${ }^{\dagger}$ Associate Professor, Department of Aerospace Engineering, University of Michigan, AIAA Associate Fellow 
morphing shapes at different flight conditions is generated using a total of 407 aerodynamic shape optimizations. Once the database is generated, we can compute the required optimal morphing shapes and related fuel burn reductions for each mission. For comparison purposes, we also perform optimizations with full morphing wings to quantify the theoretical minimal drag for each condition.

This paper is organized as the follows: Section II discusses the computational tools used in this study. The baseline geometry and optimization problem formulations are shown in Section III. We first perform a multipoint optimization of the wing in Section IV. The trailing edge morphing optimization results are presented in Section V. Then, we discuss the full morphing wing optimizations and comparison with morphing trailing edge in Section VI and Section VII. We simulate a number of flight missions and quantify the fuel burn reduction with the adaptive morphing trailing edge in Section VIII.

\section{Computational Tools}

This section describes the numerical tools and methods that are used for the shape optimization studies. These tools are components of the framework for multidisciplinary design optimization (MDO) of aircraft configurations with high fidelity (MACH) [7]. MACH can perform the simultaneous optimization of aerodynamic shape and structural sizing variables considering aeroelastic deflections [8]. However, in this paper we use only the components of MACH that are relevant for aerodynamic shape optimization: the geometric parametrization, mesh perturbation, CFD solver, and optimization algorithm. This setup has successfully been used to study aerodynamic design optimization problems [9, $6,10,11]$.

\section{A. Geometric Parametrization}

We use a free form deformation (FFD) approach to parametrize the geometry [12]. The FFD volume parametrizes the geometry changes rather than the geometry itself, resulting in a more efficient and compact set of geometry design variables, and thus making it easier to handle complex geometric manipulations. Any geometry may be embedded inside the volume by performing a Newton search to map the parameter space to physical space. All the geometric changes are performed on the outer boundary of the FFD volume. Any modification of this outer boundary indirectly modifies the embedded objects. The key assumption of the FFD approach is that the geometry has constant topology throughout the optimization process, which is usually the case for wing design. In addition, since FFD volumes are trivariate B-spline volumes, the sensitivity information of any point inside the volume can be easily computed. Figure 1 shows the FFD volume and geometric control points for the aerodynamic shape optimization.

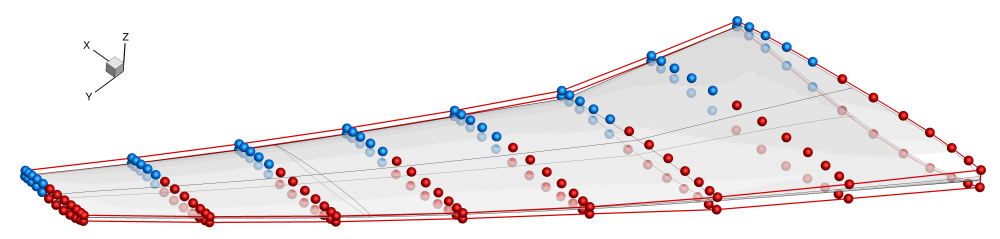

Figure 1. The wing shape design variables are the $z$-displacement of 192 FFD control points (red and blue spheres). The trailing edge morphing design variables are only the blue control points.

To simulate the trailing edge morphing, the last 5 chordwise control points (shown in blue), which correspond to the last $45 \%$ of the chord, can move independently for each flight condition, thus providing sufficient degrees-offreedom to alter the airfoil camber and spanwise twist distribution to simulate the morphing trailing edge similar to the FlexSys adpative wing [2]. Because of the constant topology assumption of the FFD approach, and due to limitations in the mesh perturbation, the surface has to be continuous around the control surfaces, eliminating the elevator gap. Therefore, when the control surfaces deflect, there is a transition region between the control surface and the centerbody, similar to those studied in a continuous morphing wing [2]. 


\section{B. Mesh Perturbation}

Since FFD volumes modify the geometry during the optimization, we must perturb the mesh for the CFD analysis to solve for the modified geometry. The mesh perturbation scheme used in this work is a hybridization of algebraic and linear elasticity methods [12]. The idea behind the hybrid warping scheme is to apply a linear-elasticity-based warping scheme to a coarse approximation of the mesh to account for large, low-frequency perturbations, and to use the algebraic warping approach to attenuate small, high-frequency perturbations. The goal is to compute a high-quality perturbed mesh similar to that obtained using a linear elasticity scheme, but at a much lower computational cost.

\section{CFD Solver}

We use the SUmb flow solver [13]. SUmb is a finite-volume, cell-centered multiblock solver for the compressible Euler, laminar Navier-Stokes, and RANS equations (steady, unsteady, and time-periodic). It provides options for a variety of turbulence models with one, two, or four equations and options for adaptive wall functions. The JamesonSchmidt-Turkel (JST) scheme [14] augmented with artificial dissipation is used for the spatial discretization. The main flow is solved using an explicit multi-stage Runge-Kutta method along with a geometric multi-grid scheme. A segregated Spalart-Allmaras (SA) turbulence equation is iterated with the diagonally dominant alternating direction implicit (DDADI) method. An automatic differentiation adjoint for the Euler and RANS equations was developed to compute the gradients $[15,6]$. The adjoint implementation supports both the full-turbulence and frozen-turbulence modes, but in the present work we use the full-turbulence adjoint exclusively. The adjoint equations are solved with preconditioned GMRES [16] using PETSc [17, 18, 19].

\section{Optimization Algorithm}

Because of the high computational cost of CFD solutions, it is critical to choose an optimization algorithm that requires a reasonably low number of function evaluations. Gradient-free methods, such as genetic algorithms, have a higher probability of getting closer to the global minimum for cases with multiple local minima. However, slow convergence and the large number of function evaluations make gradient-free aerodynamic shape optimization infeasible with the current computational resources, especially for large numbers of design variables. In addition, the local minima of this problem has very similar shapes as investigated in $[20,11]$. Since we require hundreds of design variables, we use a gradient-based optimizer combined with adjoint gradient evaluations to solve the problem efficiently.

The optimization algorithm we use for all results presented herein is SNOPT (sparse nonlinear optimizer) [21] through the Python interface pyOpt [22]. SNOPT is a gradient-based optimizer that implements a sequential quadratic programming method; it is capable of solving large-scale nonlinear optimization problems with thousands of constraints and design variables. SNOPT uses a smooth augmented Lagrangian merit function, and the Hessian of the Lagrangian is approximated using a limited-memory quasi-Newton method.

\section{Optimization Problem Formulation}

All optimization cases perform lift-constrained drag minimization of the wing using the RANS equations. In this section, we provide a complete description of the problems. The baseline optimization problem formulation has been previously used by the authors [11].

\section{A. Baseline Geometry}

The baseline geometry is a wing with a blunt trailing edge extracted from the NASA Common Research Model (CRM) wing-body geometry, which is representative of a contemporary transonic commercial transport [23, 24], with a size similar to that of a Boeing 777. Several design features, such as an aggressive pressure recovery in the outboard wing, were introduced into the design to make it more interesting for research purposes, and to protect intellectual property. This baseline geometry provides a reasonable starting point for optimization, while leaving room for further performance improvements. In addition, the CRM was designed together with the fuselage of the full CRM configuration, so its performance is degraded when only the wing is considered. 


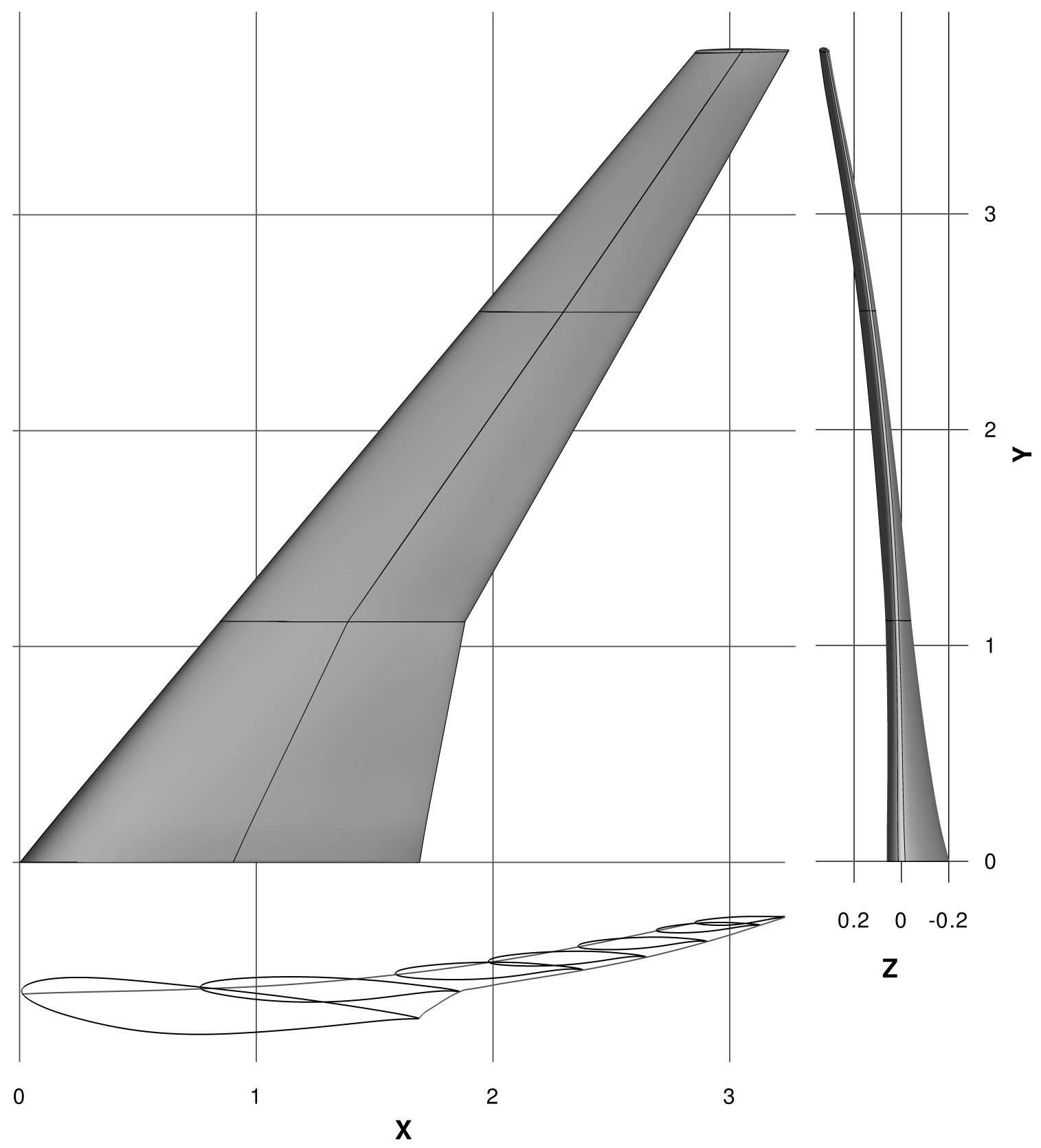

Figure 2. Baseline CRM wing geometry scaled by its mean aerodynamic chord. 
The geometry and specifications for this wing are given by the Aerodynamic Design Optimization Discussion Group (ADODG) ${ }^{\mathrm{a}}$ and were used as the basis for a benchmark single point aerodynamic shape optimization problem defined by the group [11]. The baseline geometry is shown in Fig. 2. All coordinates are scaled by the mean aerodynamic chord (275.8 in). The resulting reference chord is 1.0, and the half span is 3.758151 . The moment reference point is at $x=1.2077$ and $z=0.007669$, while the reference area is 3.407014 .

\section{B. Volume Grid}

We generate the mesh for the CRM wing using an in-house hyperbolic mesh generator. The mesh is marched out from the surface mesh using an O-grid topology to a farfield located at a distance of 25 times the span (about 185 mean chords). The nominal cruise flow condition is Mach 0.85, with a Reynolds number of 5 million based on the mean aerodynamic chord. The mesh size and the flow solution values at the nominal operating condition are listed in Table 1, and are the same as the on used by the authors to solve the ADODG CRM single-point benchmark problem [11].

\begin{tabular}{rrcccc}
\hline Mesh level & Mesh size & $\boldsymbol{C}_{\boldsymbol{D}}$ & $\boldsymbol{C}_{\boldsymbol{L}}$ & $\boldsymbol{C}_{\boldsymbol{M}}$ & $\boldsymbol{\alpha}$ \\
\hline$h=0$ & $\infty$ & 0.01990 & & & \\
L00 & $230,686,720$ & 0.01992 & 0.5000 & -0.1776 & $2.2199^{\circ}$ \\
L0 & $28,835,840$ & 0.01997 & 0.5000 & -0.1790 & $2.2100^{\circ}$ \\
L1 & $3,604,480$ & 0.02017 & 0.5000 & -0.1810 & $2.1837^{\circ}$ \\
L2 & 450,560 & 0.02111 & 0.5000 & -0.1822 & $2.1944^{\circ}$ \\
\hline
\end{tabular}

Table 1. Mesh convergence study for the baseline CRM wing[11].

We perform a mesh convergence study to determine the resolution accuracy of this mesh. Table 1 lists the drag and moment coefficients for the baseline meshes. We also compute the zero-grid spacing drag using Richardson's extrapolation, which estimates the drag value as the grid spacing approaches zero [25]. The zero-grid spacing drag coefficient is 199.0 counts for the baseline CRM wing. The surface and symmetry plane mesh for the L0, L1, and L2 grid levels are shown in Fig. 3. Since we need to perform hundreds of optimizations to optimize the trailing edge for each flight conditions, we use the L2 mesh to achieve a reasonable computational cost with sufficient accuracy. For simplicity, we only use the L2 mesh for the studies in this paper. However, a multilevel approach could be used to optimize a larger grid size [26].

\section{Objective Function}

The baseline multipoint aerodynamic shape optimization seeks to minimize averaged drag coefficients by varying the shape design variables subject to constraints on the lift, pitching moment, and maneuver bending moment. The drag coefficients are given by the RANS solutions. The drag coefficients of 5 flight conditions is considered as shown in Table 2. The bending moment constraint is computed at a $2.5 \mathrm{~g}$ maneuver condition (15,000 ft and Mach 0.86). The formulation is different from the ADODG benchmark case. A similar mulitpoint has been presented by the authors [11].

\begin{tabular}{rrr}
\hline Flow Case & $\boldsymbol{C}_{\boldsymbol{L}}$ & Mach number \\
\hline 1 & 0.50 & 0.85 \\
2 & 0.55 & 0.85 \\
3 & 0.45 & 0.85 \\
4 & 0.50 & 0.84 \\
5 & 0.50 & 0.86 \\
5 & $2.5 \mathrm{~g}$ & 0.86 \\
\hline
\end{tabular}

Table 2. The multiple flight conditions represent a five-point stencil in Mach- $C_{L}$ space and a $2.5 \mathrm{~g}$ maneuver case.

\footnotetext{
ahttps://info.aiaa.org/tac/ASG/APATC/AeroDesignopt-DG/default.aspx, last accessed May 10, 2014
} 


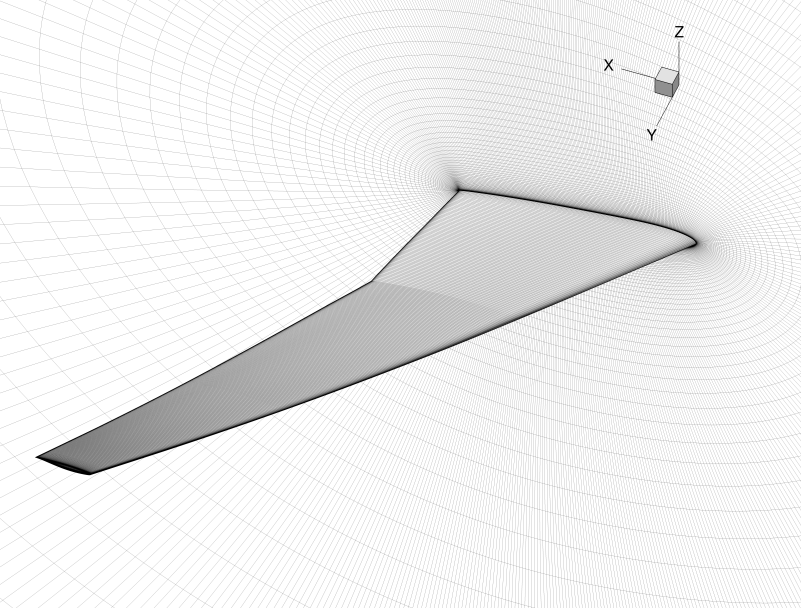

a) L0 mesh: $28.8 \mathrm{M}$ cells, 199.7 drag counts.

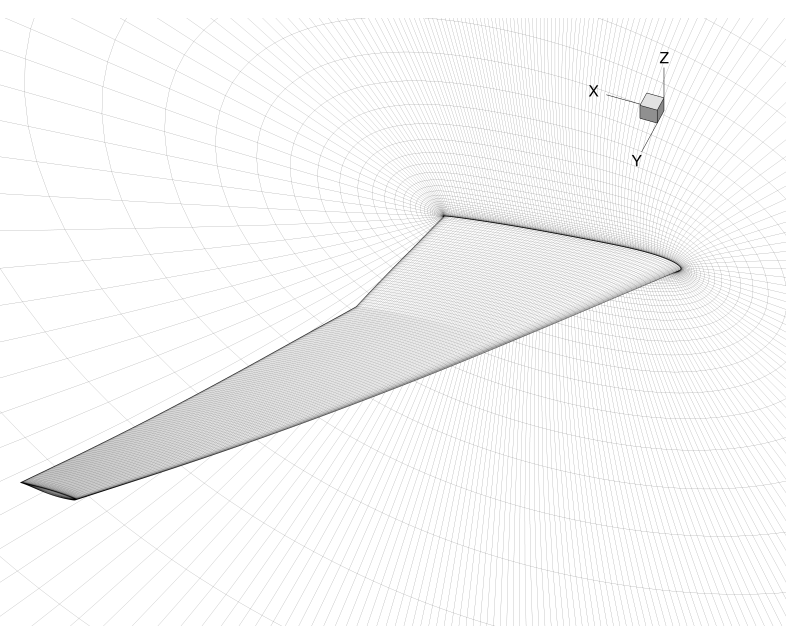

b) L1 mesh: 3.6 M cells, 201.7 drag counts.

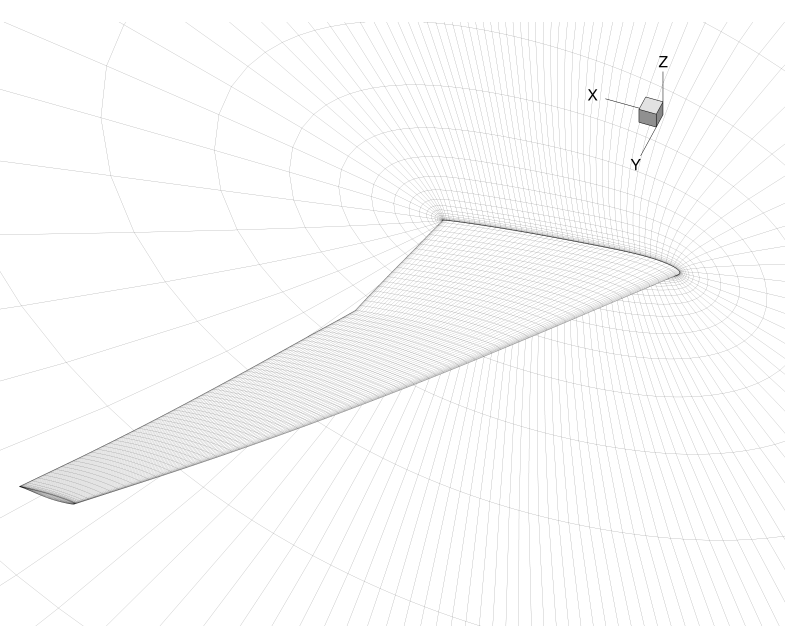

c) L2 mesh: $450 \mathrm{k}$ cells, 211.1 drag counts.

Figure 3. O-grids of varying sizes were generated using an hyperbolic mesh generator[11]. 


\section{Design Variables}

Before we study the trailing edge morphing, we performed a multipoint aerodynamic shape optimization of the wing to obtain an optimized aerodynamic performance of the wing itself. The first set of design variables consists of control points distributed on the FFD volume. A total of 192 shape variables are distributed on the lower and upper surfaces of the FFD volume, as shown in Fig. 1. The large number of shape variables provides more degrees of freedom for the optimizer to explore, and this allows us to fine-tune the sectional airfoil shapes and the thickness-to-chord ratios at each spanwise location. Because of the efficient adjoint implementation, the cost of computing the shape gradients is nearly independent of the number of shape variables [7]. The full morphing wing optimization uses the same set of shape design variables.

For the morphing trailing edge optimization, we use a subset of the shape control point near the TE as the design variables, as shown in blue in Figure 1. Only the shape on the last $45 \%$ of the chord is allowed to change. The shape of the forward wing remains constant.

\section{E. Constraints}

Since optimizers tend to exploit any weaknesses in numerical models and problem formulations, an optimization problem needs to be carefully constrained in order to yield a physically feasible design. We performed a multipoint optimization with 6 flight conditions: 5 cruise conditions and a $2.5 \mathrm{~g}$ maneuver condition. Both lift and pitching moment are constrained at the nominal flight condition (Mach 0.85, $C_{L}=0.5$ ). In addition, the wing root bending moment is constrained to be less or equal than the nominal value at the $2.5 \mathrm{~g}$ maneuver condition. We also implement several geometric constraints. First, we impose constant thickness constraints from the $1 \%$ chord at the LE to the $99 \%$ chord near the TE. A total of 750 thickness constraints are imposed in the 25 by 30 grid. The constraints have a lower bound of $100 \%$ of the baseline thickness and no upper bound. These constraints ensure sufficient height in the centerbody cabin and sufficient fuel volume. The LE thickness constraint allows for the installation of slats, and the TE thickness is limited due to manufacturing constraints. The total volume of the wing is also constrained to meet a fuel volume requirement. The complete optimization problem is described in Table 3.

\begin{tabular}{clll} 
& Function/variable & Description & Quantity \\
\hline minimize & $C_{D}$ & Drag coefficient & \\
& & Angle of attack & 1 \\
with respect to & $\alpha$ & FFD control point $z$-coordinates & 192 \\
& $z$ & Total design variables & 193 \\
& & Lift coefficient constraint & 6 \\
subject to & $C_{L}=C_{L}^{*}$ & Moment coefficient constraint & 1 \\
& $C_{M_{y}} \geq-0.17$ & Bending moment coefficient constraint & 1 \\
& $C_{b e n d} \geq C_{\text {bend }}^{*}$ & Minimum thickness constraints & 750 \\
& $t \geq t_{\text {base }}$ & Minimum volume constraint & 1 \\
& $V \geq V_{\text {base }}$ & Total constraints & 759
\end{tabular}

Table 3. Aerodynamic shape optimization problem

\section{Baseline Multipoint Aerodynamic Shape Optimization of the Wing}

Before we perform any morphing trailing edge optimization, we first optimize the wing itself using a multipoint formulation to achieve a reasonable performance that is robust with respect to different flight conditions. In this section, we present our aerodynamic design optimization results for the wing (described in Table 3) under the 5 flight conditions and a $2.5 \mathrm{~g}$ maneuver condition. We use the $\mathrm{L} 2$ grid $(450 \mathrm{k}$ cells) for the optimization. Transport aircraft operate at multiple cruise conditions because of variability in the flight missions and air traffic control restrictions. Single-point optimization at the nominal cruise condition could overstate the benefit of the optimization, since the optimization improves the on-design performance to the detriment of the off-design performance. The single-point 
optimization benchmark problem developed by the ADODG resulted in an optimal wing with an unrealistically sharp leading edge in the outboard section of the wing [11]. This was caused by a combination of the low value for the thickness constraints (25\% of the baseline) and the single-point formulation [20,11]. Therefore, in this study, we use multipoint formulation and $100 \%$ thickness constraints, which we have found to result in more realistic wings [11].

We choose five equally weighted flight conditions with different combinations of lift coefficient and Mach number, as previously done by the authors $[8,11]$. The flight conditions are the nominal cruise, $\pm 10 \%$ of cruise $C_{L}$, and \pm 0.01 of cruise Mach, as shown in Table 2. More sophisticated ways of choosing multipoint flight conditions and their associated weights can be used, such as the automated procedure developed by Liem et al. [27] that minimizes fleetlevel fuel burn. The objective function is the average drag coefficient for the five flight conditions, and the moment constraint is enforced only for the nominal flight condition. The bending moment constraint is enforced at the $2.5 \mathrm{~g}$ maneuver condition at $15,000 \mathrm{ft}$ and Mach 0.86 .
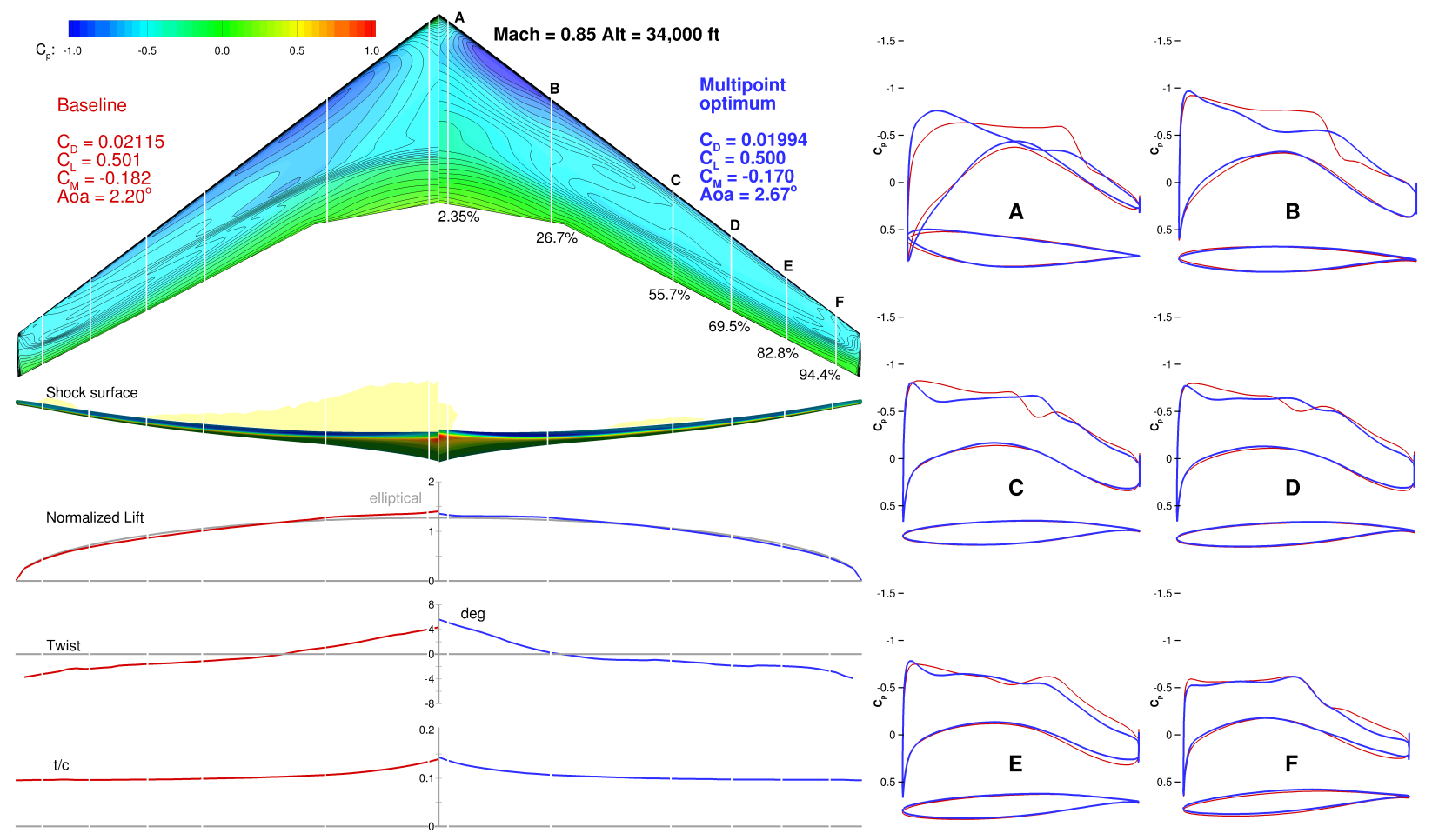

Figure 4. The multipoint optimized wing has $5.7 \%$ lower drag.

A comparison of the initial wing and multipoint optimized design is shown in Figure 4. The baseline results are shown in red, and the multipoint results are shown in blue. The $C_{p}$ for the multipoint optimized result corresponds to the nominal condition (Mach $0.85, C_{L}=0.5$ ). We compute the shock surface from the volume solution grid by constructing an isosurface of the normal Mach number [28]. The shock occurs where the normal Mach number is one, i.e.,

$$
M_{n}=\frac{\vec{u}}{a} \cdot \frac{\nabla p}{|\nabla p|}=1 .
$$

Unlike the shock-free design obtained with single-point optimization [11], the multipoint optimization settled on an optimal compromise between the flight conditions, resulting in a weak shock at all conditions. Similar trends were observed in the multipoint optimization of Vassberg et al. [29]. Our optimization procedure reduced the drag from 211.5 counts to 199.4 counts, i.e., an $5.7 \%$ reduction. At the optimum, the lift coefficient target is met, and the pitching moment is reduced to the lowest allowed value. The $2.5 \mathrm{~g}$ bending moment constraint is met. The lift distribution of the optimized wing is much closer to the elliptical distribution than that of the baseline, indicating an induced drag 
that is close to the theoretical minimum for planar wakes. This is achieved by fine-tuning the twist distribution and airfoil shapes. The baseline wing has a near-linear twist distribution. The optimized design has more twist at the root and tip, and less twist near mid-wing. This multipoint optimized wing provides a reasonable baseline geometry for the morphing trailing edge optimization.

\section{Morphing Trailing Edge Optimization}

We perform a series of RANS-based aerodynamic shape optimizations to examine the effects of trailing edge morphing. A gradient-based optimizer is used with sensitivities computed by the adjoint method. The full turbulence adjoint used includes the linearization of both the main flow solver and the SA turbulence model. The optimizations are converged to an optimality tolerance of $\mathcal{O}\left(10^{-5}\right)$. We use the optimized geometry from the previous optimization in Section IV as the baseline.

The shape aft of the $45 \%$ chord is free to change independently for each flight condition. The airfoil thickness is kept constant by the thickness constraints. A total of 80 design variables are used for each optimization. The angleof-attack is also allowed to change during the optimization. To span the entire flight envelope, we performed 407 separate optimizations at various altitudes, Mach numbers, and weights. Each optimization required about 4 hours on 64 processors, corresponding to about 50 optimization iterations. No additional moment constraints are imposed in the optimization. Since the TE can be morphed at each flight condition, the $2.5 \mathrm{~g}$ maneuver bending moment constraints can be satisfied using the deflected TE. Therefore, we can check to see whether the $2.5 \mathrm{~g}$ bending moment constraint can be satisfied independently from the TE optimization at cruise conditions. Figures 5 to 8 show the trailing edge optimization results at several on- and off-design conditions. Results for additional flight conditions are shown in Figures 14 to 27.

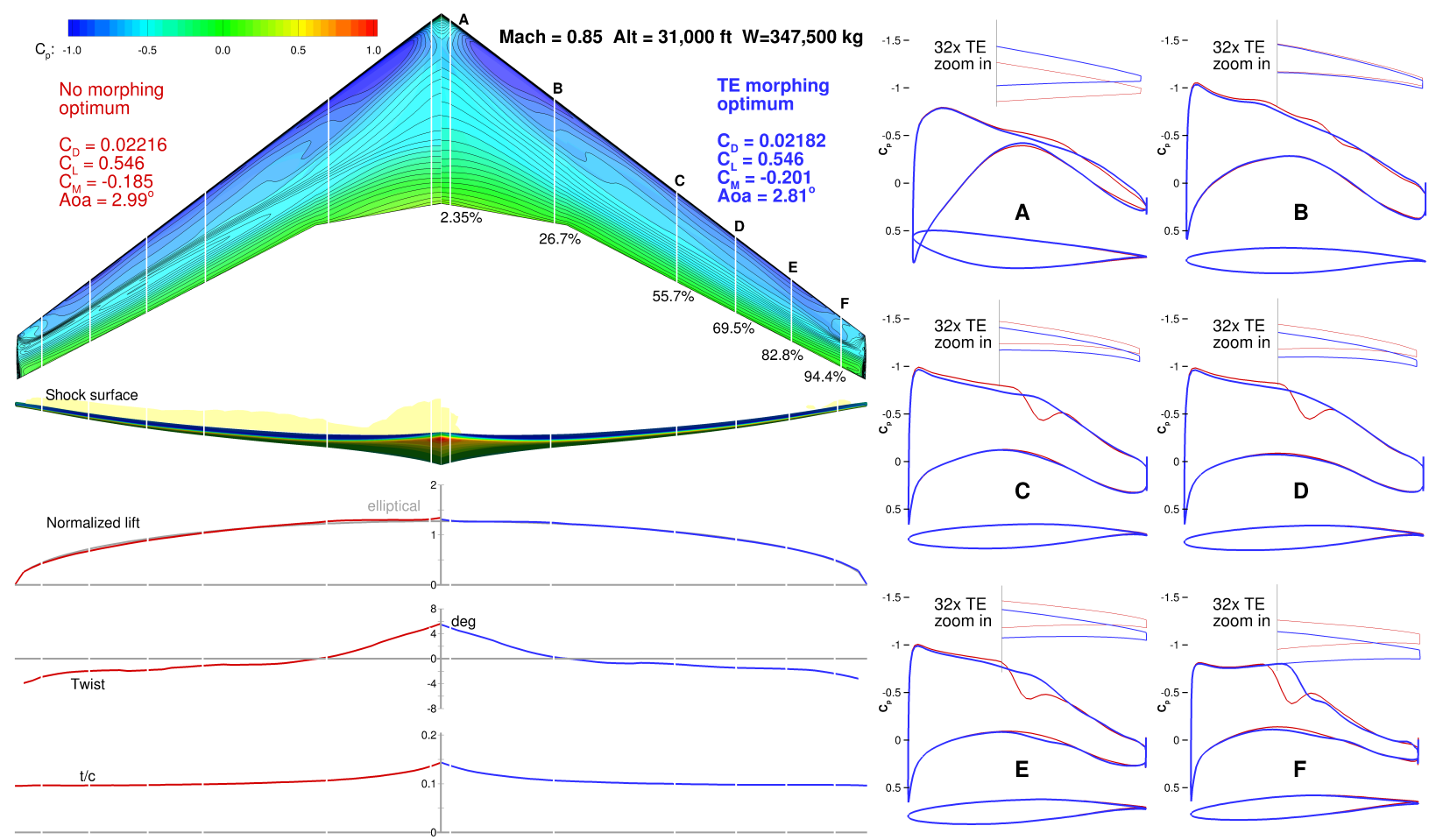

Figure 5. Morphing trailing edge optimization at MTOW on-design condition.

At on-design conditions, the drag reductions range from 1 to $2 \%$. The optimized TE shapes are extremely close to the initial shape. However, we see that the optimizer is able to further smooth out the flow by introducing a slight camber at the TE. The TE deflection is less than 1 degree (measured from LE). The shock strength is reduced, 


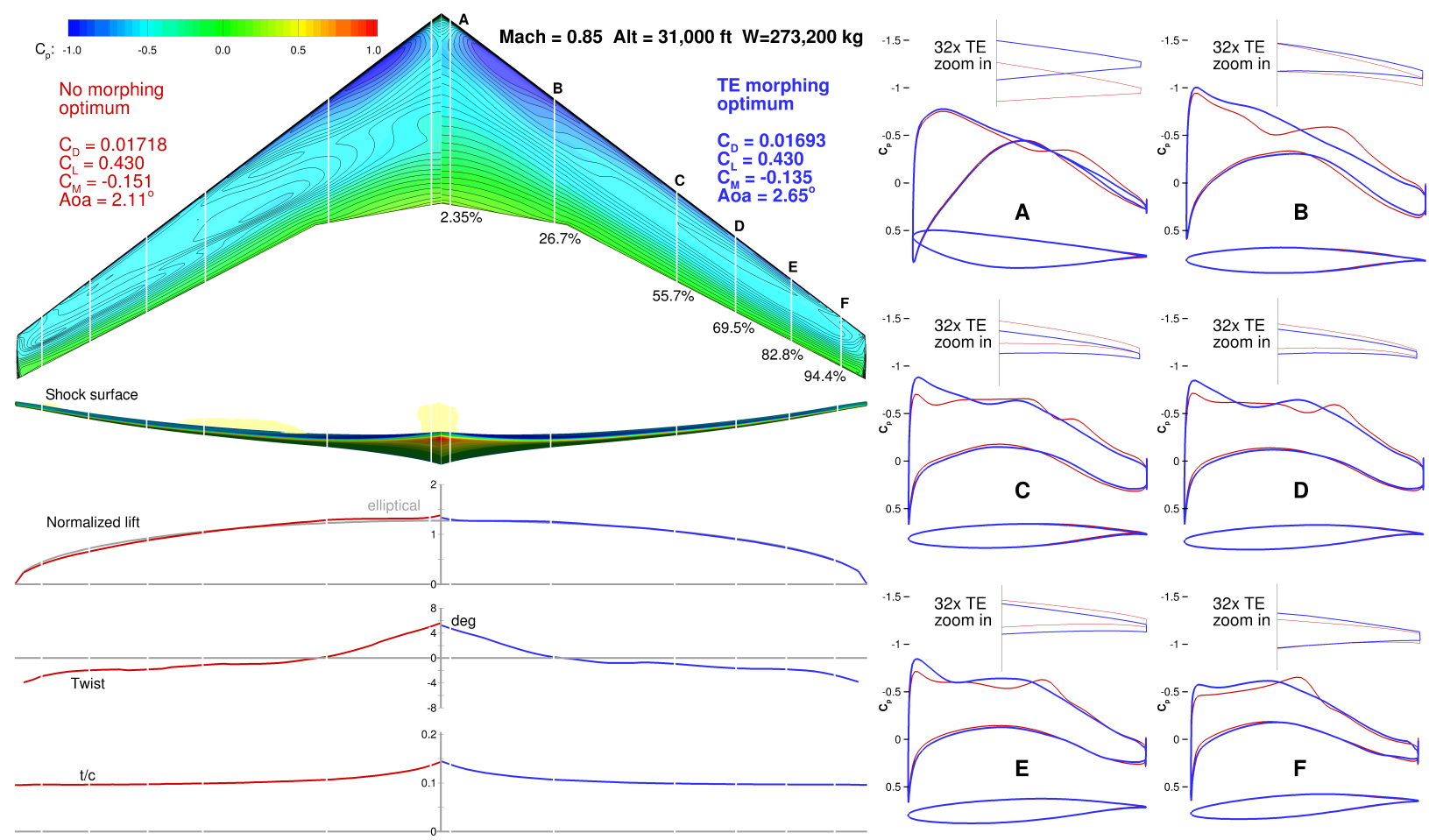

Figure 6. Morphing trailing edge optimization at half-weight on-design condition.
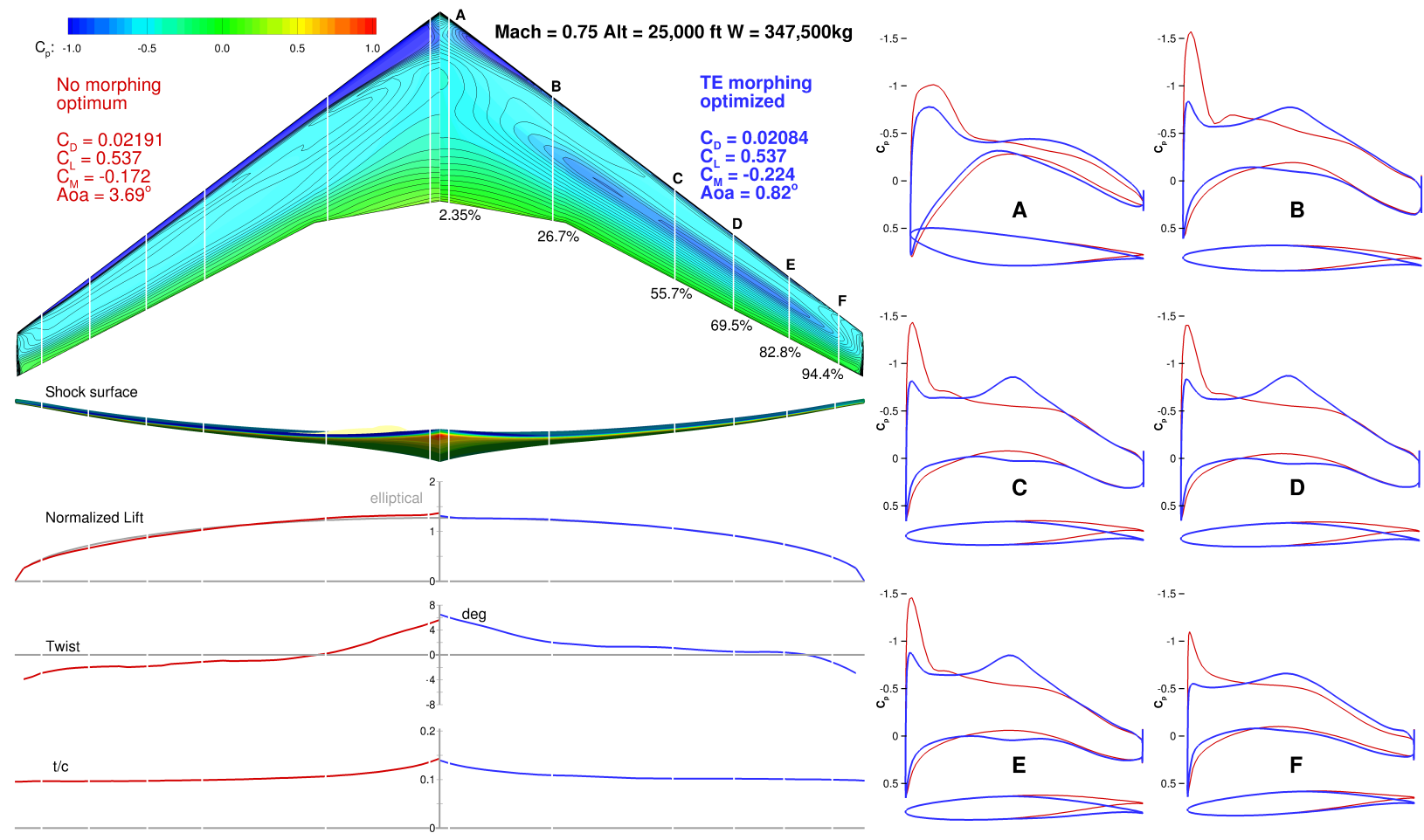

Figure 7. Morphing trailing edge optimization at low-Mach low-altitude off-design condition. 


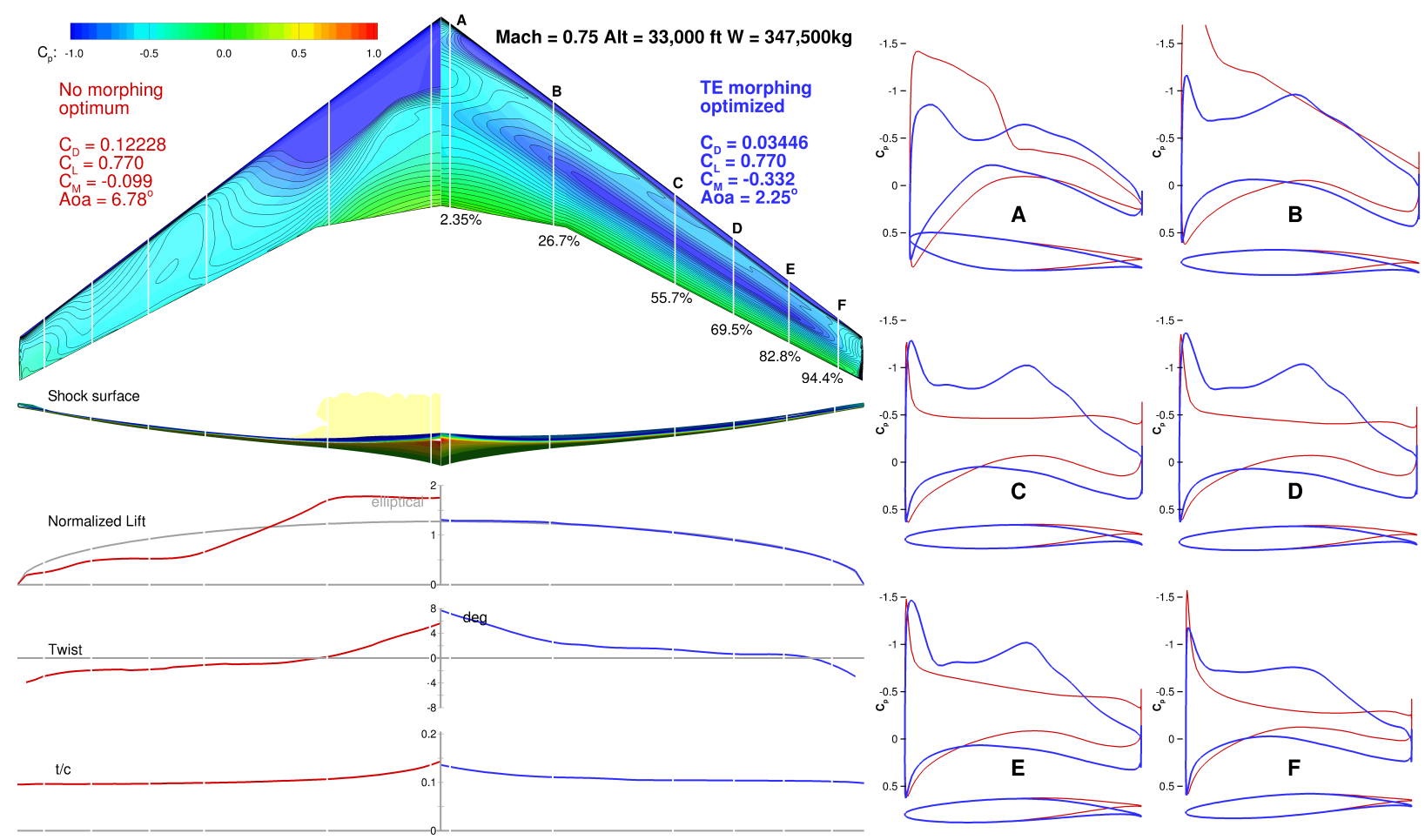

Figure 8. Morphing trailing edge optimization at low-Mach high-altitude off-design condition.

illustrating that the transonic flow is sensitive to even slight changes in trailing edge shape. Similar trends are observed at several different weights.

At the off-design conditions, the difference between the optimized TE shape and the initial TE shape is more apparent. The maximum TE deflection at off-design conditions is about 3 degrees. The drag reduction due to morphing TE is more significant, reaching 5\%. At some extreme off-design cases, the flow is separated without a morphing TE. By optimizing the TE camber and shape, the angle-of-attack is reduced and the flow is re-attached, significantly reducing the drag. The $2.5 \mathrm{~g}$ maneuver bending moment constraint is satisfied using the TE morphing. We conclude that the drag at all flight conditions can be reduced using morphing TE, and that the benefit of a morphing trailing edge is more significant at off-design conditions.

\section{Full Wing Morphing Optimization}

We also performed a shape optimization assuming a fully morphing wing. While the technology for achieving such morphing is currently not available, we are interested in finding out how much the performance would increase relative to the morphing TE. A total of 192 design variables are used to optimize the entire wing at each flight condition. The rest of the optimization setup is the same as that in Section V. Similarly, to span the entire flight envelope, we performed another 407 separate optimizations for different altitudes, Mach numbers, and weights. Due to the increased design space, the computational cost of the optimization is slightly higher: 6 hours on 64 processors instead of 4 hours in the TE morphing case. Figures 9 and 10 show the full morphing wing optimization results for an on-design and an off-design condition.

At on-design conditions, the full wing morphing designs are only marginally better than the optimized design with morphing TE. Specifically, the drag coefficient is decreased by about 1 count. The baseline wing is already optimized near the cruise conditions. Additional drag reduction is difficult to achieve even with a full morphing wing. The optimized wing shapes are very close to the initial shape. The pressure distributions are also quite similar to that of the morphing TE optimized designs. Therefore, we see that it is sufficient to only change the TE shape for drag reduction purpose at on-design conditions. 


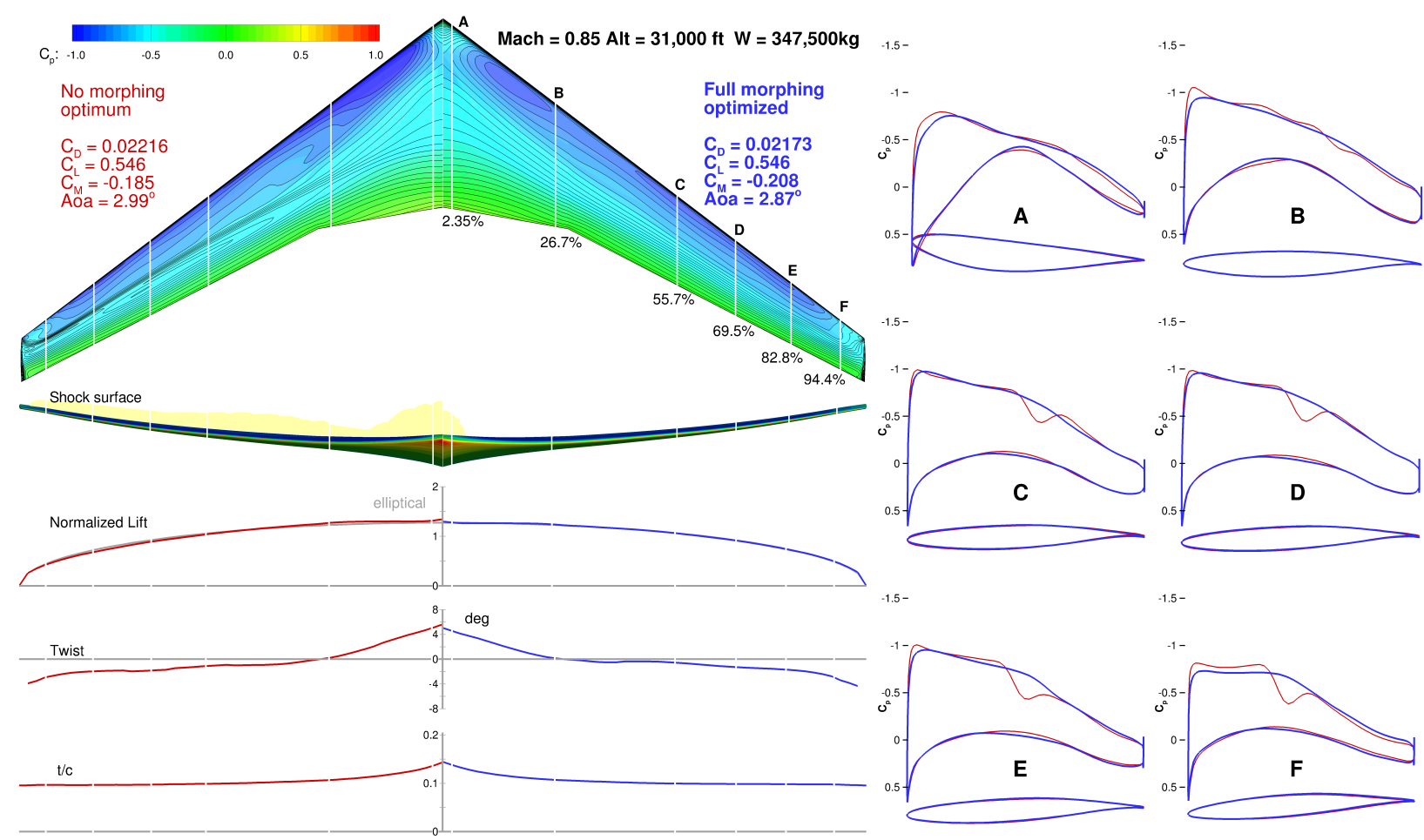

Figure 9. Full wing morphing optimization at MTOW on-design condition.
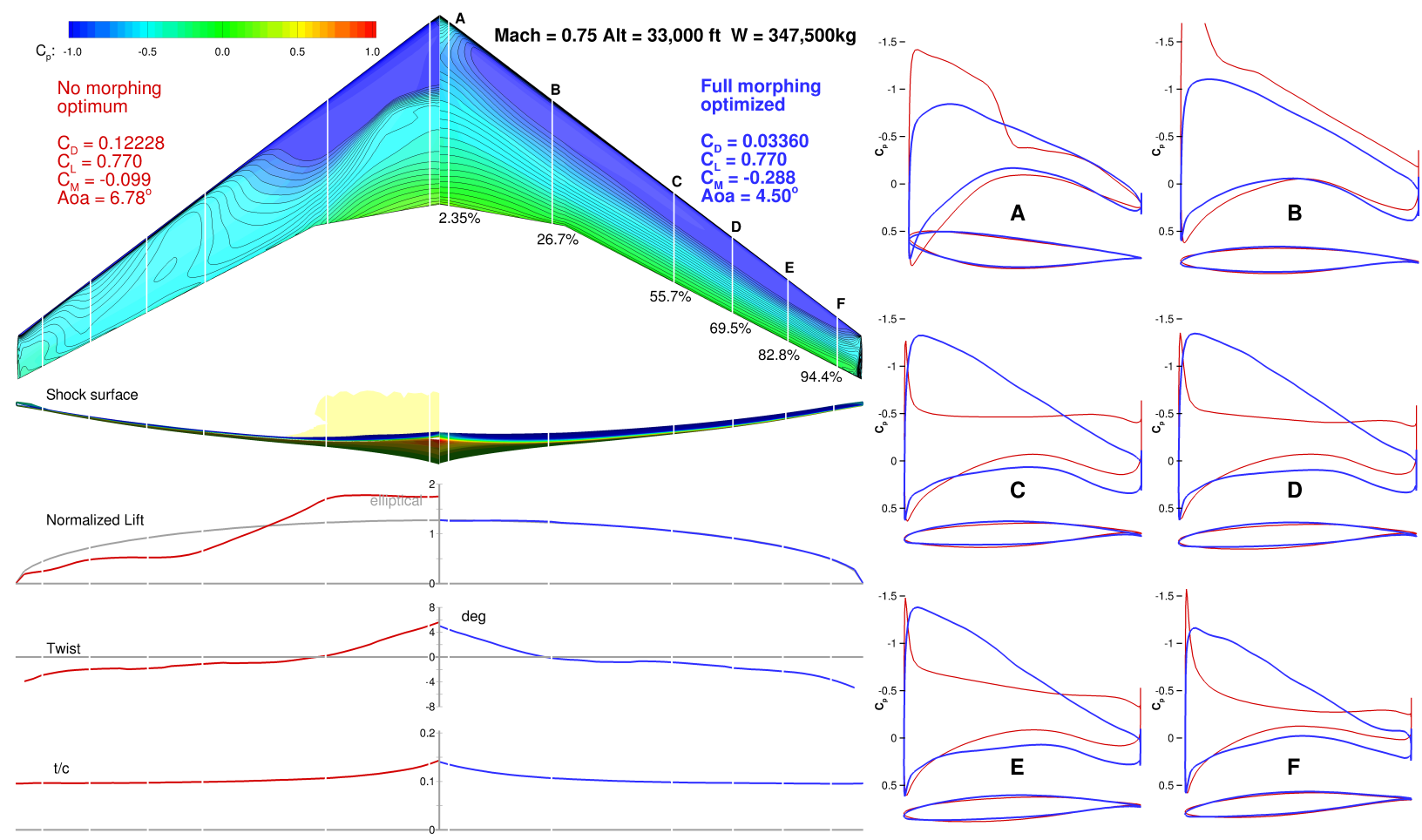

Figure 10. Full wing morphing optimization at low-Mach high-altitude off-design condition. 
At the off-design conditions, additional improvements of up to 10 drag counts are achieved. The maximum TE deflection at off-design conditions is about 3 degrees. The drag reduction due to morphing TE is much more significant at more than 5\%. In the flight condition shown in Figure 10, the flow on the initial wing is separated. The full morphing wing still maintains a shock-free solution and near-elliptical lift distribution even at high $C_{L}$. We observe that the benefit of morphing wing can be magnified at off-design conditions.

\section{Comparison between Morphing Trailing Edge and Fully Morphing Wing}

To further compare the benefits of the morphing TE and the morphing wing, we plotted the percentage drag reduction contours of each approach for the entire flight envelope for MTOW (347,500 kg), as shown in Figure 11. The drag reduction contours for other weights are shown in Figures 28 and 29. The weight and altitude range is based on the Boeing 777-200LR operation manual for Long Range Cruise (LRC).

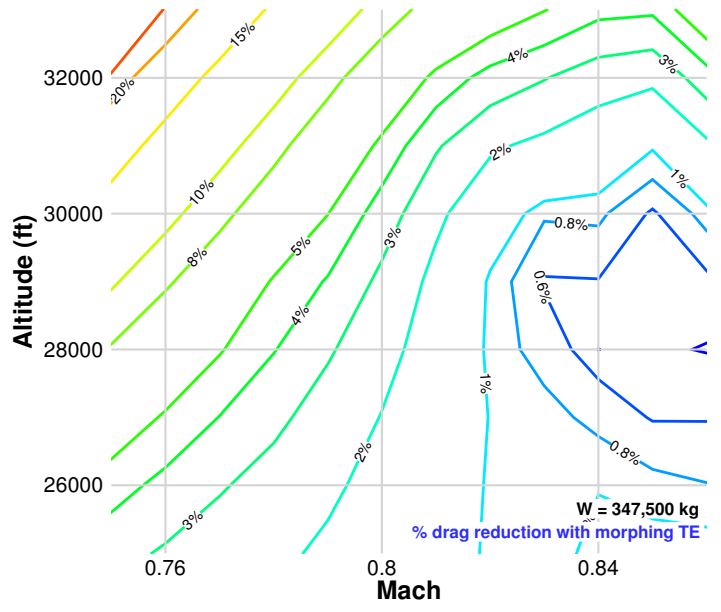

a) Morphing TE

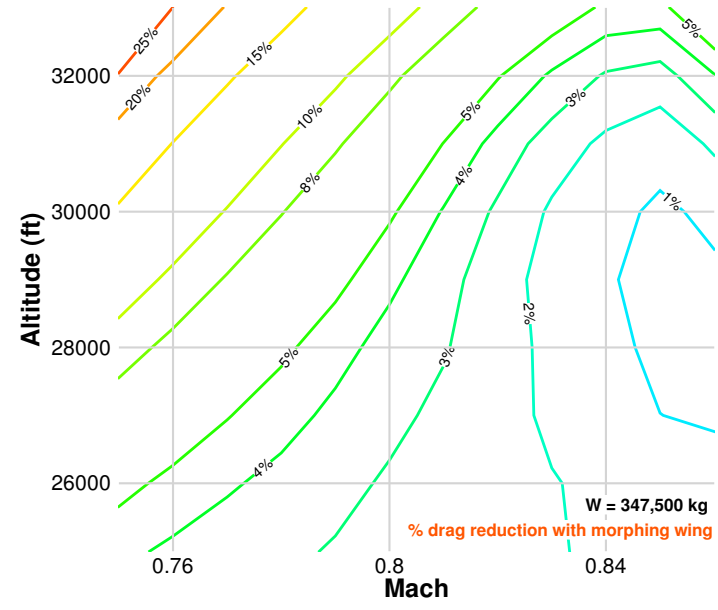

b) Morphing Wing

Figure 11. The trends of the drag reduction contours are similar, with a drag reduction of about $1 \%$ near on-design conditions.

The trends of both drag reduction contours are similar. The lowest drag reductions are near the on-design conditions where the wing has been previously optimized with a multipoint formulation. Those drag reductions are due to the additional degrees-of-freedom that allow the TE shape to change separately at each flight condition, and to making the $2.5 \mathrm{~g}$ maneuver condition constraint independent through load alleviation with the morphing TE. At the lower Mach number range, the drag reduction increases with the altitude and Mach number. The highest drag reduction occurred at the flight condition with high altitude and low Mach, where the lift coefficient is the highest. For high Mach numbers above 0.85 , the trend reverses due to the drag divergence.

We also plot $M L / D$ contours of the multipoint baseline, morphing TE, and fully morphing wing designs with respect to altitude and Mach number in Figure 12. $M L / D$ contours for other weights are shown in Figures 30 and 31. $M L / D$ provides a metric for quantifying aircraft range based on the Breguet range equation with constant thrust-specific fuel consumption. While the thrust-specific fuel consumption is actually not constant, assuming it to be constant is acceptable when comparing performance in a limited Mach number range [30]. We add 100 drag counts to the computed drag to account for the drag due to the fuselage, tail, and nacelles, and we get more realistic $M L / D$ values. In aircraft design, the $99 \%$ value of the maximum $M L / D$ contour, shown in black, is often used to examine the robustness of the design [23]. The point with the highest Mach number on that contour line corresponds to the long range cruise (LRC) point, which is the point at which the aircraft can fly at a higher speed by incurring a $1 \%$ increase in fuel burn [31]. 


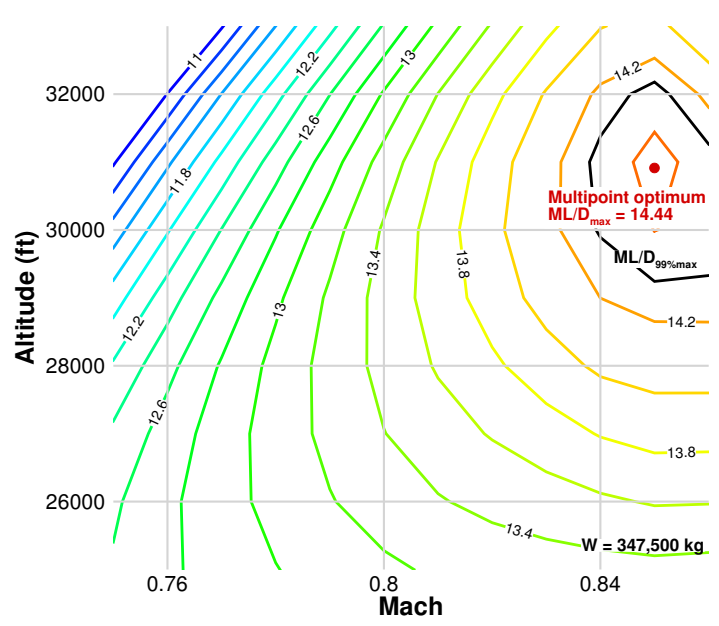

a) Multipoint optimized baseline

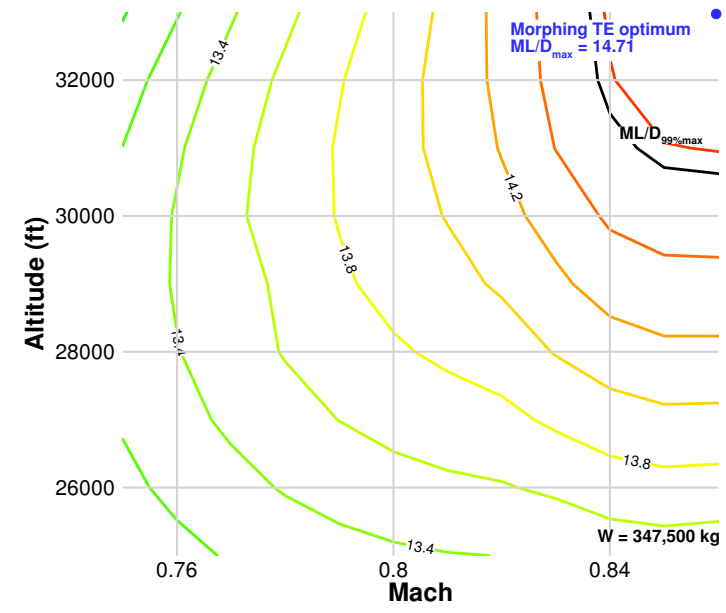

b) Morphing TE

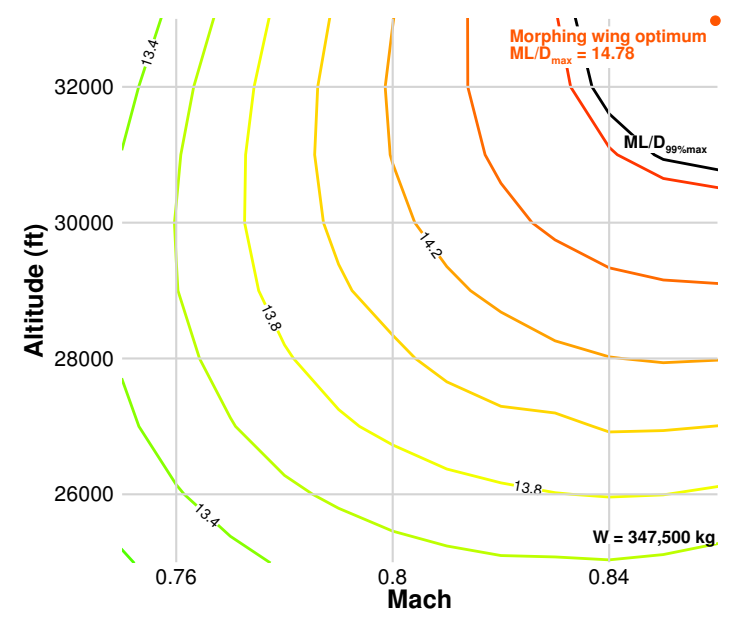

c) Morphing Wing

Figure 12. The maximum $M L / D$ occurs at a higher altitude and Mach number with morphing

The multipoint baseline maximum $M L / D$ occurs at the nominal flight condition (Mach $0.85,31,000 \mathrm{ft}$ altitude). Both morphing TE and morphing wing increase the maximum $M L / D$. The maximum $M L / D$ points for morphing TE and morphing wing are at a higher altitude and higher Mach number. Since the TE shape can be adapted for each flight condition, the drag divergence is pushed to a higher Mach number. The $99 \%$ value of the maximum $M L / D$ contour of the morphing designs are also significantly enlarged, indicating a more robust design. We see that morphing TE enables aircraft to fly higher and faster without a fuel burn penalty. To more accurately capture the tradeoffs, a multidisciplinary study including low speed aerodynamics, propulsion, and structure, would be required. 


\section{Simulating Flights with Morphing Trailing Edge}

Since we have morphing trailing edge optimizations spanning the entire flight envelope, we can create a surrogate model of optimal trailing edge shapes for different flight conditions. This database allows us to compute the fuel burn for a series of missions without performing any additional optimizations. Since we have a relatively fine discretization of the flight region, we use a linear interpolation to evaluate the performance and optimal shape between the optimized points. A thrust specific fuel consumption (TSFC) of $0.53 \mathrm{lb} /(\mathrm{lbf} \cdot \mathrm{h}$ ) is assumed. We also add 100 drag counts to the computed drag to account for the drag due to the fuselage, tail, and nacelles. The fuel burn is then integrated backwards for a given flight profile. Figure 13 shows a typical flight profile for a long range flight (currently the longest non-stop commercial flight from Dallas Fort Worth to Sydney, Australia).

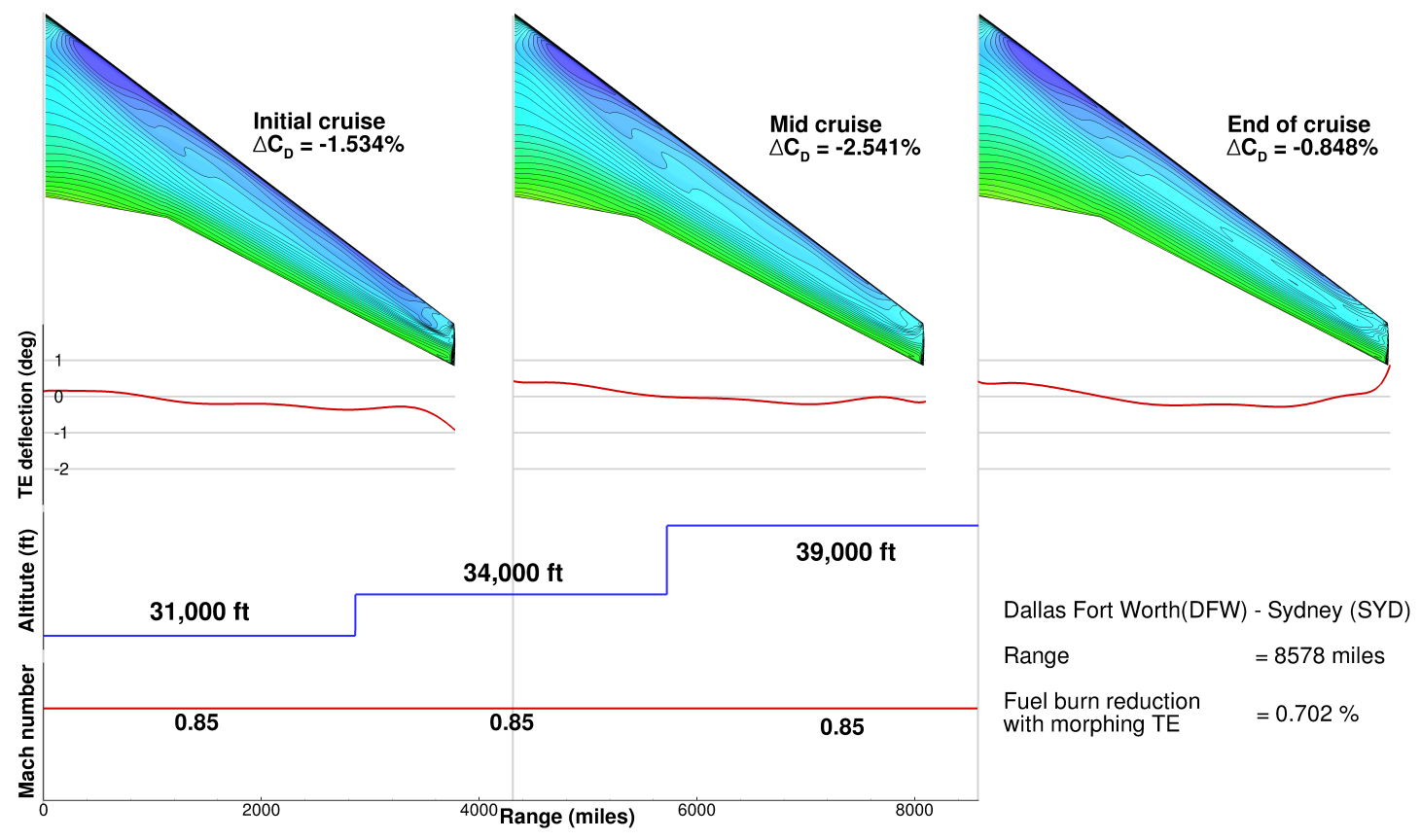

Figure 13. Fuel burn is reduced by $0.7 \%$ using morphing TE for DFW-SYD flight.

Since the flight is operated in the on-design condition with step climb, the trailing edge deflection is within 1 degree. The wing tip exhibits the highest amount of deflection with -1 degree at the initial cruise to 1 degree near the end of the cruise. We see a $0.7 \%$ fuel burn reduction using morphing TE on this flight. As pointed out in Section V, the morphing TE has higher drag reduction at off-design conditions. Table 4 shows the drag reduction on a number of hypothetical flight trajectories.

\begin{tabular}{rrrrrr}
\hline Departure & Arrival & Distance (miles) & Mach number & Cruise Altitude (feet) & Fuel burn reduction \\
\hline DFW & SYD & 8578 & $0.85,0.85,0.85$ & $31,000,34,000,39,000$ & $-0.702 \%$ \\
DTW & PVG & 7120 & $0.85,0.85,0.85$ & $33,000,36,000,41,000$ & $-1.023 \%$ \\
LAX & NRT & 5440 & $0.80,0.85,0.80$ & $33,000,33,000,33,000$ & $-1.049 \%$ \\
JFK & SFO & 2580 & $0.84,0.84,0.84$ & $32,000,34,000,36,000$ & $-1.207 \%$ \\
ATL & ORD & 606 & $0.78,0.80,0.78$ & $31,000,31,000,31,000$ & $-1.074 \%$ \\
- & - & 2000 & $0.75,0.75,0.75$ & $29,000,31,000,34,000$ & $-1.68 \%$ \\
\hline
\end{tabular}

Table 4. The fuel burn reduction is about $1 \%$ using morphing TE for various flight trajectories. 
We see that the morphing TE provides about $1 \%$ fuel burn reduction at cruise condition for the simulated flights in Table 4. All of the simulated flights have TE deflection within 2 degrees. Additional benefits could be realized during the climb and descent, which is neglected in this analysis. To evaluate the climb and descent, additional optimizations at lower speeds and lower altitudes would be needed to span the flight envelope for climb and descent.

\section{Conclusions}

We presented the aerodynamic shape optimization of a Boeing 777-size wing with an adaptive morphing trailing edge. A mulitpoint optimization, including a $2.5 \mathrm{~g}$ maneuver condition, was presented to provide a baseline for the TE optimization. A total of 407 trailing edge optimizations with different Mach number, altitude, and weight, were performed to span the entire cruise flight envelope. A drag reduction in the order of $1 \%$ is achieved for on-design conditions, and reductions up to 5\% were achieved for off-design conditions.

We further evaluated the performance of a morphing trailing edge by comparing its benefits with those from a full morphing wing. This is done by plotting the drag reduction contour and the $M L / D$ contour. The full morphing wing yielded only marginally lower drag and a similar $M L / D$ contour. Therefore, morphing only the TE can achieve an aerodynamic performance similar to that of a fully morphing wing without the drastic increase in wing morphing mechanism and weight.

Finally, we created a surrogate model of optimal trailing edge shapes to compute cruise fuel burn for different flight missions. We observed about $1 \%$ fuel burn reduction using the morphing trailing edge. More significant fuel burn reduction could be achieved in climb and descent segments.

From an aerodynamic perspective, an adaptive morphing trailing edge can easily offer additional drag reduction without a complete redesign of the wing. Since this technology has been demonstrated by FlexSys, and could be installed on conventional control surfaces, we could consider retrofitting existing aircraft. To thoroughly evaluate the benefit, a multidisciplinary study is required to examine the trade-offs between aerodynamics, structures, and controls.

\section{Acknowledgments}

The computations were performed on the Flux HPC cluster at the University of Michigan Center of Advanced Computing, and on the Gordon cluster of the Extreme Science and Engineering Discovery Environment (XSEDE), which is supported by National Science Foundation grant number ACI-1053575. This research is partially funded by FlexSys, Inc. The authors would like to thank Yin Yu for his assistance in generating figures, as well as our colleagues at MDOlab for numerous ideas.

\section{References}

[1] Kota, S., Hetrick, J., Osborn, R., Paul, D., Pendleton, E., Flick, P., and Tilmann, C., "Design and Application of Compliant Mechanisms for Morphing Aircraft Structures," Proceedings of SPIE, Vol. 5054, 2003, p. 25.

[2] Kota, S., Osborn, R., Ervin, G., Maric, D., Flick, P., and Paul, D., "Mission Adaptive Compliant Wing-Design, Fabrication and Flight Test," RTO Applied Vehicle Technology Panel (AVT) Symposium, 2009.

[3] Sofla, A. Y. N., Meguid, S. A., Tan, K. T., and Yeo, W. K., "Shape Morphing of Aircraft Wing: Status and Challenges," Materials \& Design, Vol. 31, No. 3, 3 2010, pp. 1284-1292. doi:10.1016/j.matdes.2009.09.011.

[4] Molinari, G., Quack, M., Dmitriev, V., Morari, M., Jenny, P., and Ermanni, P., "Aero-Structural Optimization of Morphing Airfoils for Adaptive Wings," Journal of Intelligent Material Systems and Structures, Vol. 22, No. 10, 2011, pp. 1075-1089. doi:10.1177/1045389X11414089.

[5] Lee, D., Gonzalez, L. F., Periaux, J., and Onate, E., "Robust Aerodynamic Design Optimisation of Morphing Aerofoil/Wing using Distributed MOGA," Proceedings of the 28th Congress of the International Council of the Aeronautical Sciences, Optimage Ltd, on behalf of the International Council of the Aeronautical Sciences (ICAS)., 2012.

[6] Lyu, Z., Kenway, G., Paige, C., and Martins, J. R. R. A., "Automatic Differentiation Adjoint of the Reynolds-Averaged Navier-Stokes Equations with a Turbulence Model," 43rd AIAA Fluid Dynamics Conference and Exhibit, June 2013.

[7] Kenway, G. K. W., Kennedy, G. J., and Martins, J. R. R. A., "Scalable Parallel Approach for High-Fidelity SteadyState Aeroelastic Analysis and Adjoint Derivative Computations," AIAA Journal, Vol. 52, No. 5, 2014, pp. 935-951. doi:10.2514/1.J052255. 
[8] Kenway, G. K. W. and Martins, J. R. R. A., "Multipoint High-fidelity Aerostructural Optimization of a Transport Aircraft Configuration,” Journal of Aircraft, Vol. 51, No. 1, 2014, pp. 144-160. doi:10.2514/1.C032150.

[9] Lyu, Z. and Martins, J. R. R. A., “Aerodynamic Shape Optimization of a Blended-Wing-Body Aircraft," 51st AIAA Aerospace Sciences Meeting including the New Horizons Forum and Aerospace Exposition, 2013. doi:10.2514/6.2013-283.

[10] Lyu, Z. and Martins, J. R. R. A., "Aerodynamic Design Optimization Studies of a Blended-Wing-Body Aircraft," Journal of Aircraft, 2014. doi:10.2514/1.C032491.

[11] Lyu, Z., Kenway, G. K. W., and Martins, J. R. R. A., "Aerodynamic Shape Optimization Investigations of the Common Research Model Wing Benchmark," AIAA Journal, 2014, (Submitted).

[12] Kenway, G. K., Kennedy, G. J., and Martins, J. R. R. A., "A CAD-free Approach to High-Fidelity Aerostructural Optimization," Proceedings of the 13th AIAA/ISSMO Multidisciplinary Analysis Optimization Conference, Fort Worth, TX, 2010. doi:10.2514/6.2010-9231.

[13] van der Weide, E., Kalitzin, G., Schluter, J., and Alonso, J., "Unsteady Turbomachinery Computations Using Massively Parallel Platforms," 44th AIAA Aerospace Sciences Meeting and Exhibit, 2006. doi:10.2514/6.2006-421.

[14] Jameson, A., Schmidt, W., and Turkel, E., "Numerical Solution of the Euler equations by Finite Volume Methods Using Runge Kutta Time Stepping Schemes," 14th AIAA, Fluid and Plasma Dynamics Conference, 1981.

[15] Mader, C. A., Martins, J. R. R. A., Alonso, J. J., and van der Weide, E., “ADjoint: An Approach for the Rapid Development of Discrete Adjoint Solvers,” AIAA Journal, Vol. 46, No. 4, April 2008, pp. 863-873. doi:10.2514/1.29123.

[16] Saad, Y. and Schultz, M. H., "GMRES: A Generalized Minimal Residual Algorithm for Solving Nonsymmetric Linear Systems," SIAM Journal on Scientific and Statistical Computing, Vol. 7, No. 3, 1986, pp. 856-869. doi:10.1137/0907058.

[17] Balay, S., Gropp, W. D., McInnes, L. C., and Smith, B. F., "Efficient Management of Parallelism in Object Oriented Numerical Software Libraries," Modern Software Tools in Scientific Computing, edited by E. Arge, A. M. Bruaset, and H. P. Langtangen, Birkhäuser Press, 1997, pp. 163-202. doi:10.1007/978-1-4612-1986-6_8.

[18] Balay, S., Brown, J., , Buschelman, K., Eijkhout, V., Gropp, W. D., Kaushik, D., Knepley, M. G., McInnes, L. C., Smith, B. F., and Zhang, H., "PETSc Users Manual,” Tech. Rep. ANL-95/11 - Revision 3.4, Argonne National Laboratory, 2013.

[19] Balay, S., Brown, J., Buschelman, K., Gropp, W. D., Kaushik, D., Knepley, M. G., McInnes, L. C., Smith, B. F., and Zhang, H., "PETSc Web page," 2013, http://www.mcs.anl.gov/petsc.

[20] Lyu, Z., Kenway, G. K. W., and Martins, J. R. R. A., "RANS-based Aerodynamic Shape Optimization Investigations of the Common Research Model Wing," AIAA Science and Technology Forum and Exposition (SciTech), National Harbor, MD, January 2014.

[21] Gill, P. E., Murray, W., and Saunders, M. A., "SNOPT: An SQP Algorithm for Large-Scale Constrained Optimization,” SIAM journal on optimization, Vol. 12, No. 4, 2002, pp. 979-1006. doi:10.1137/S1052623499350013.

[22] Perez, R. E., Jansen, P. W., and Martins, J. R. R. A., "pyOpt: A Python-Based Object-Oriented Framework for Nonlinear Constrained Optimization," Structures and Multidisciplinary Optimization, Vol. 45, No. 1, 2012, pp. 101-118. doi:10.1007/s00158-011-0666-3.

[23] Vassberg, J., Dehaan, M., Rivers, M., and Wahls, R., "Development of a Common Research Model for Applied CFD Validation Studies," 26th AIAA Applied Aerodynamics Conference, American Institute of Aeronautics and Astronautics, August 2008. doi:10.2514/6.2008-6919.

[24] Vassberg, J., "A Unified Baseline Grid about the Common Research Model Wing/Body for the Fifth AIAA CFD Drag Prediction Workshop (Invited)," 29th AIAA Applied Aerodynamics Conference, Jul 2011. doi:10.2514/6.2011-3508.

[25] Roache, P. J., "Verification of Codes and Calculations," AIAA Journal, Vol. 36, No. 5, 1998, pp. 696-702. doi:10.2514/2.457.

[26] Lyu, Z. and Martins, J. R. R. A., "Strategies for Solving High-Fidelity Aerodynamic Shape Optimization Problems," AIAA Aviation and Aeronautics Forum and Exposition (AVIATION 2014), Atlanta, GA, June 2014.

[27] Liem, R. P., Kenway, G. K. W., and Martins, J. R. R. A., "Multi-mission Aircraft Fuel Burn Minimization via Multi-point Aerostructural Optimization,” AIAA Journal, 2014, (Submitted).

[28] Haimes, R., "Automated Feature Extraction from Transient CFD Simulations," Proceeding of the 7th Annual Conference of the CFD Society of Canada, Halifax, NS, May 1999.

[29] Vassberg, J. and Jameson, A., "Influence of Shape Parameterization on Aerodynamic Shape Optimization," Tech. rep., Von Karman Institute, Brussels, Belgium, April 2014.

[30] Torenbeek, E., Advanced Aircraft Design: Conceptual Design, Technology and Optimization of Subsonic Civil Airplanes, Aerospace Series, Wiley, 2013. doi:10.1002/9781118568101.

[31] Roberson, W., Root, R., and Adams, D., "Fuel Conservation Stragtergies: Cruise Flight,” Tech. rep., Boeing AERO Magazine, 2007. 

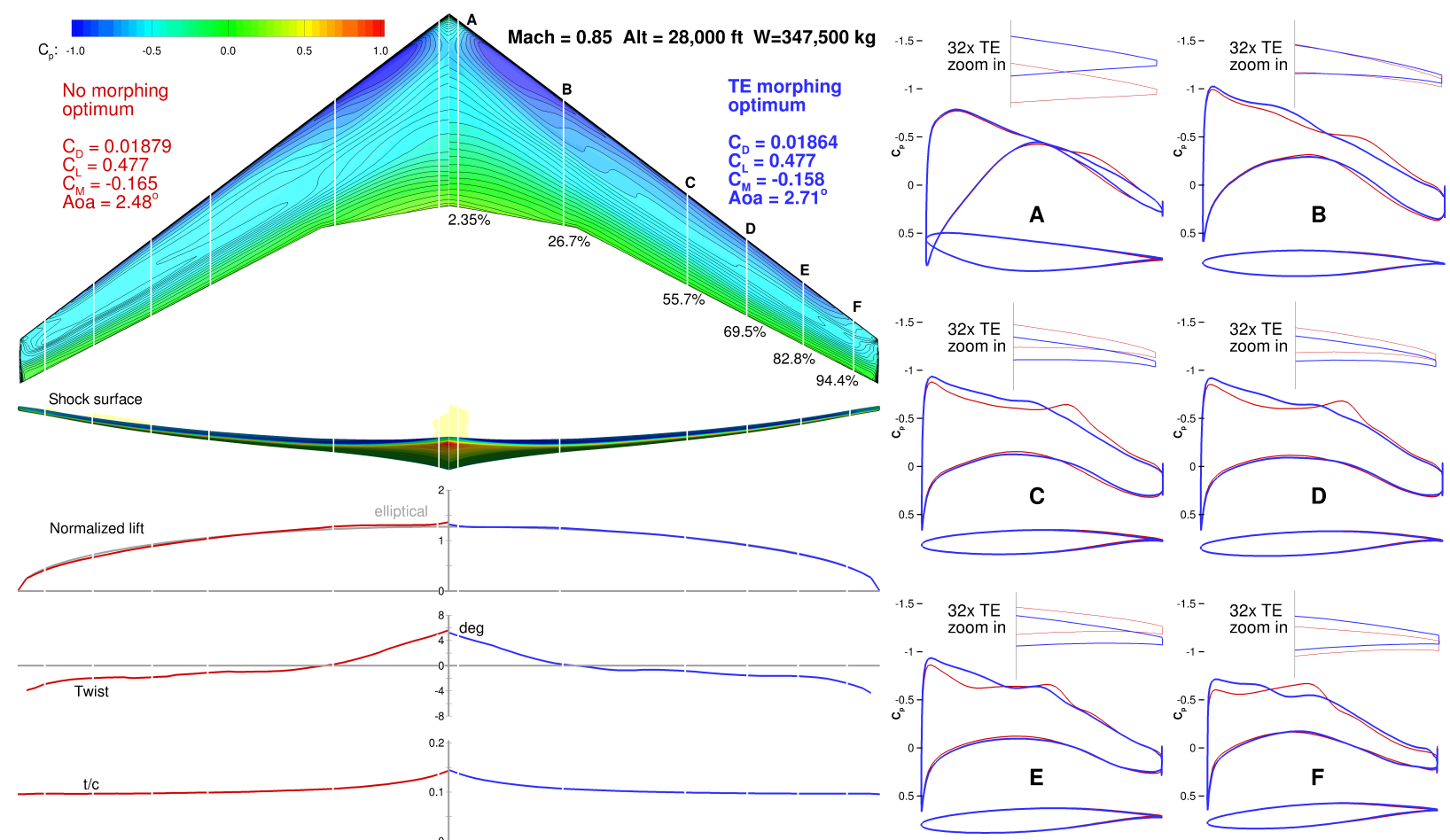

Figure 14. Morphing trailing edge optimization at $\mathrm{M}=\mathbf{0 . 8 5}$, Alt $=28,000 \mathrm{ft}$, and $\mathrm{W}=347,500 \mathrm{~kg}$.
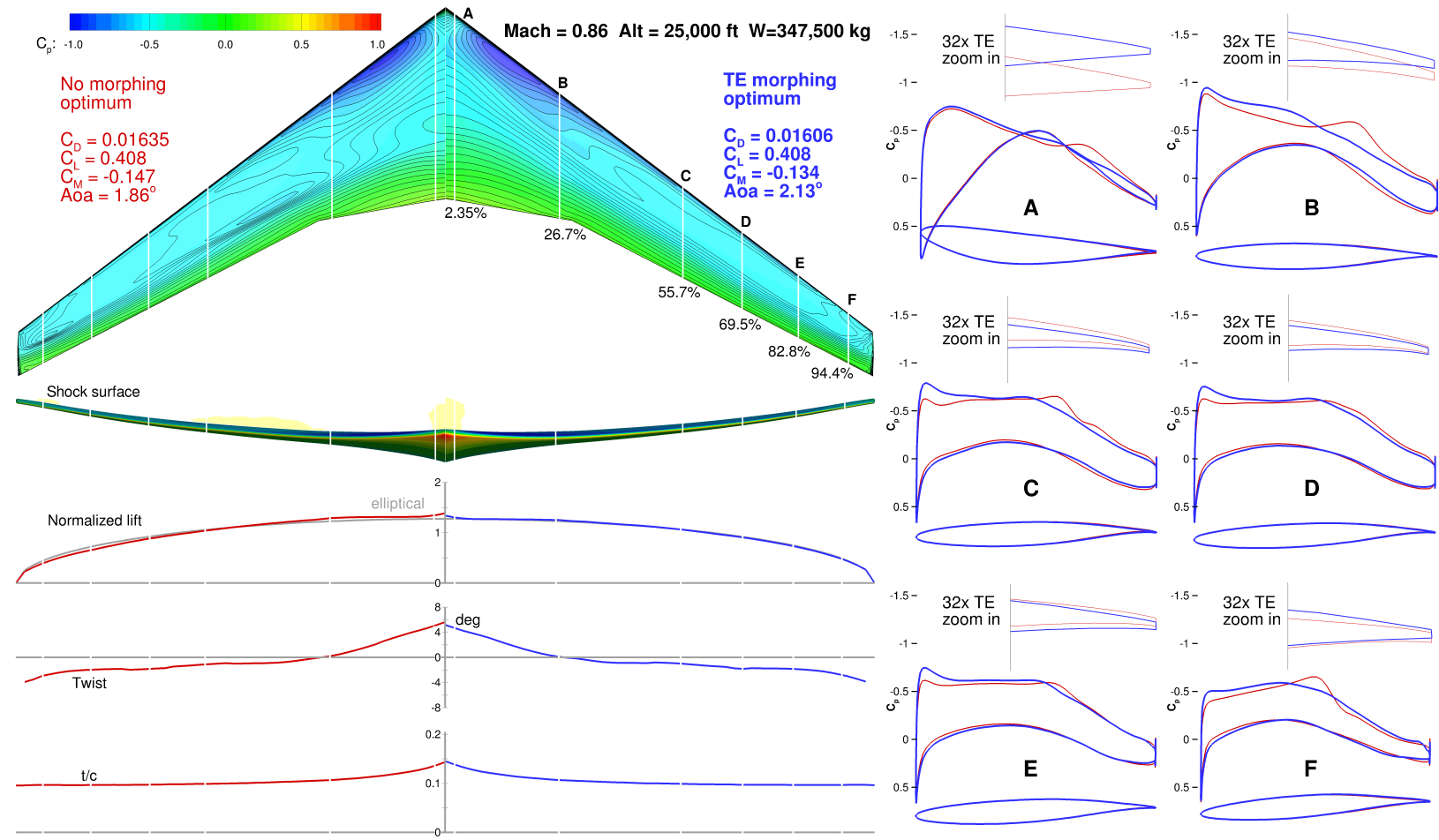

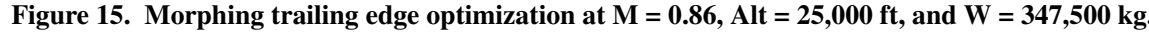



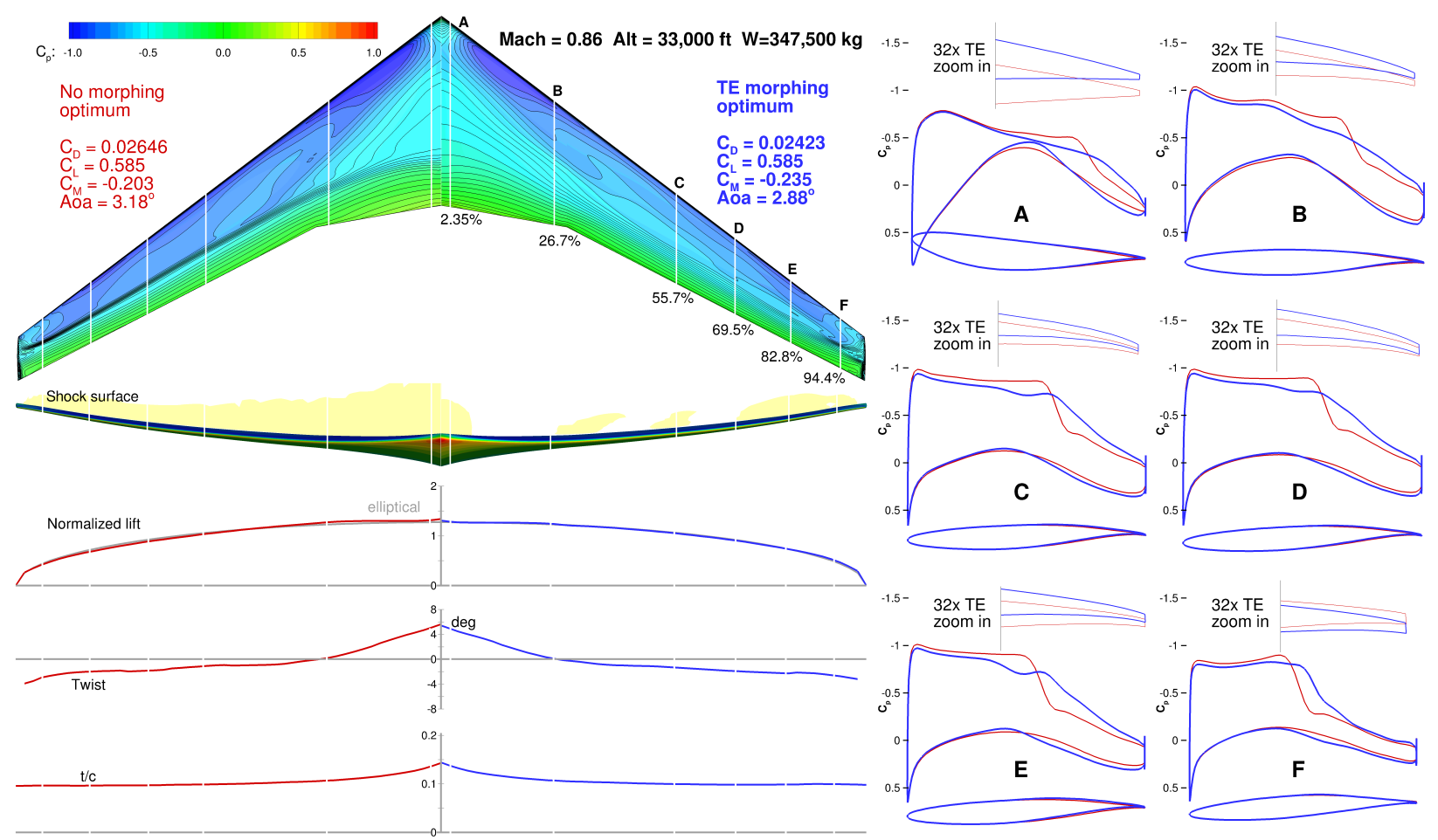

Figure 16. Morphing trailing edge optimization at $\mathrm{M}=\mathbf{0 . 8 6}$, $\mathrm{Alt}=33,000 \mathrm{ft}$, and $\mathrm{W}=347,500 \mathrm{~kg}$.
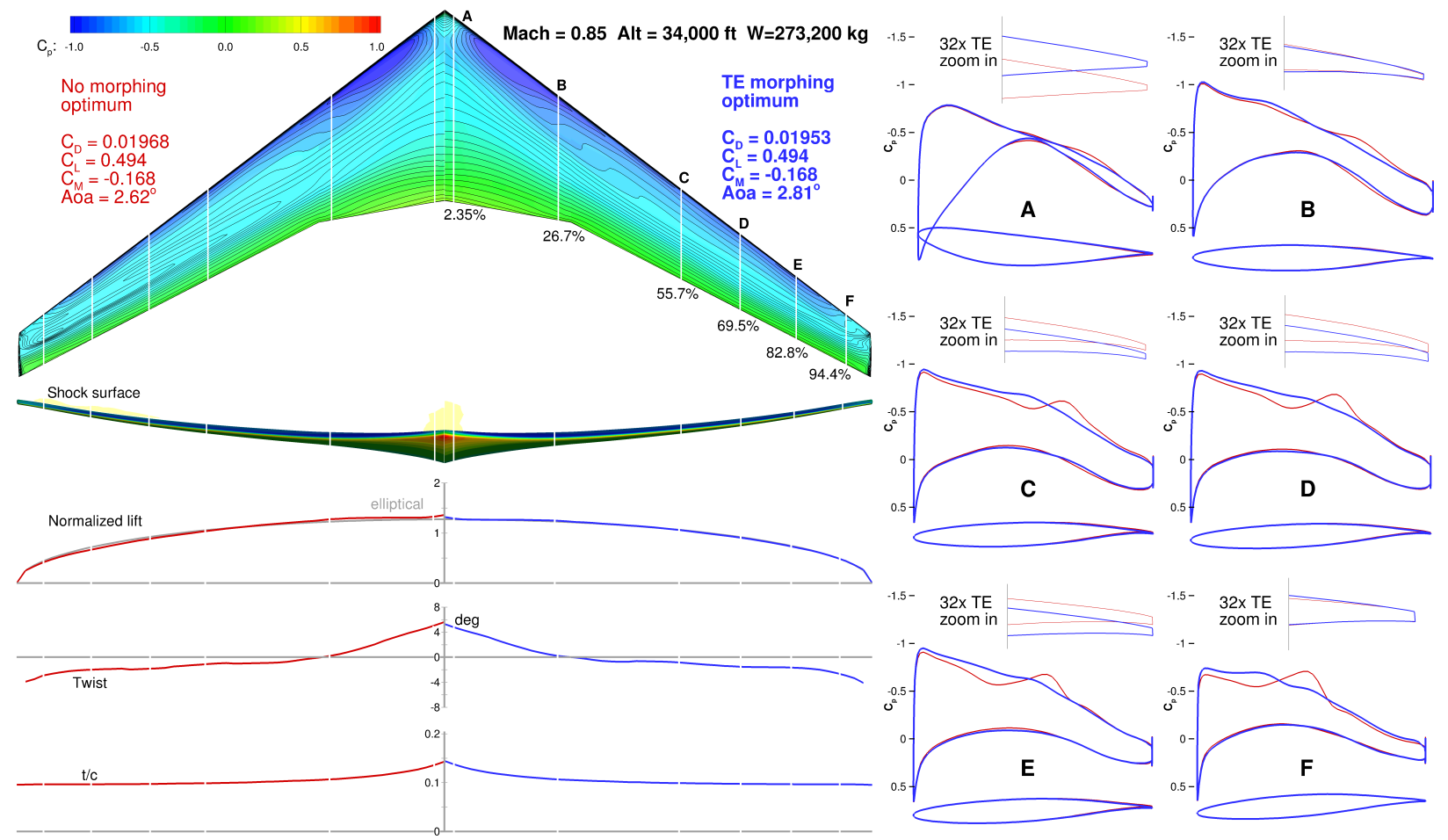

Figure 17. Morphing trailing edge optimization at $M=0.85$, Alt $=34,000 \mathrm{ft}$, and $\mathrm{W}=\mathbf{2 7 3 , 2 0 0} \mathrm{kg}$. 

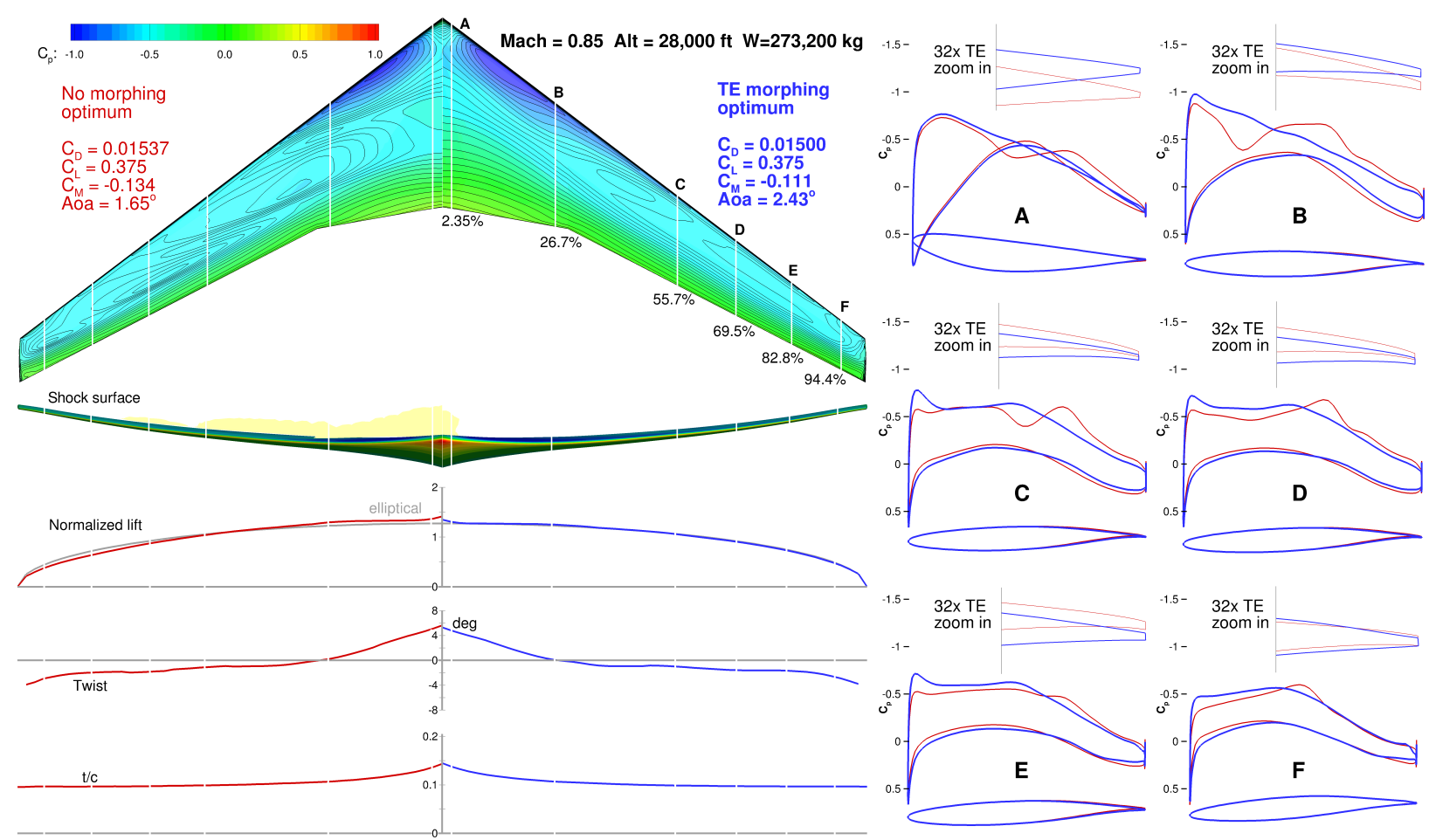

Figure 18. Morphing trailing edge optimization at $M=0.85$, Alt $=28,000 \mathrm{ft}$, and $\mathrm{W}=\mathbf{2 7 3 , 2 0 0} \mathrm{kg}$.
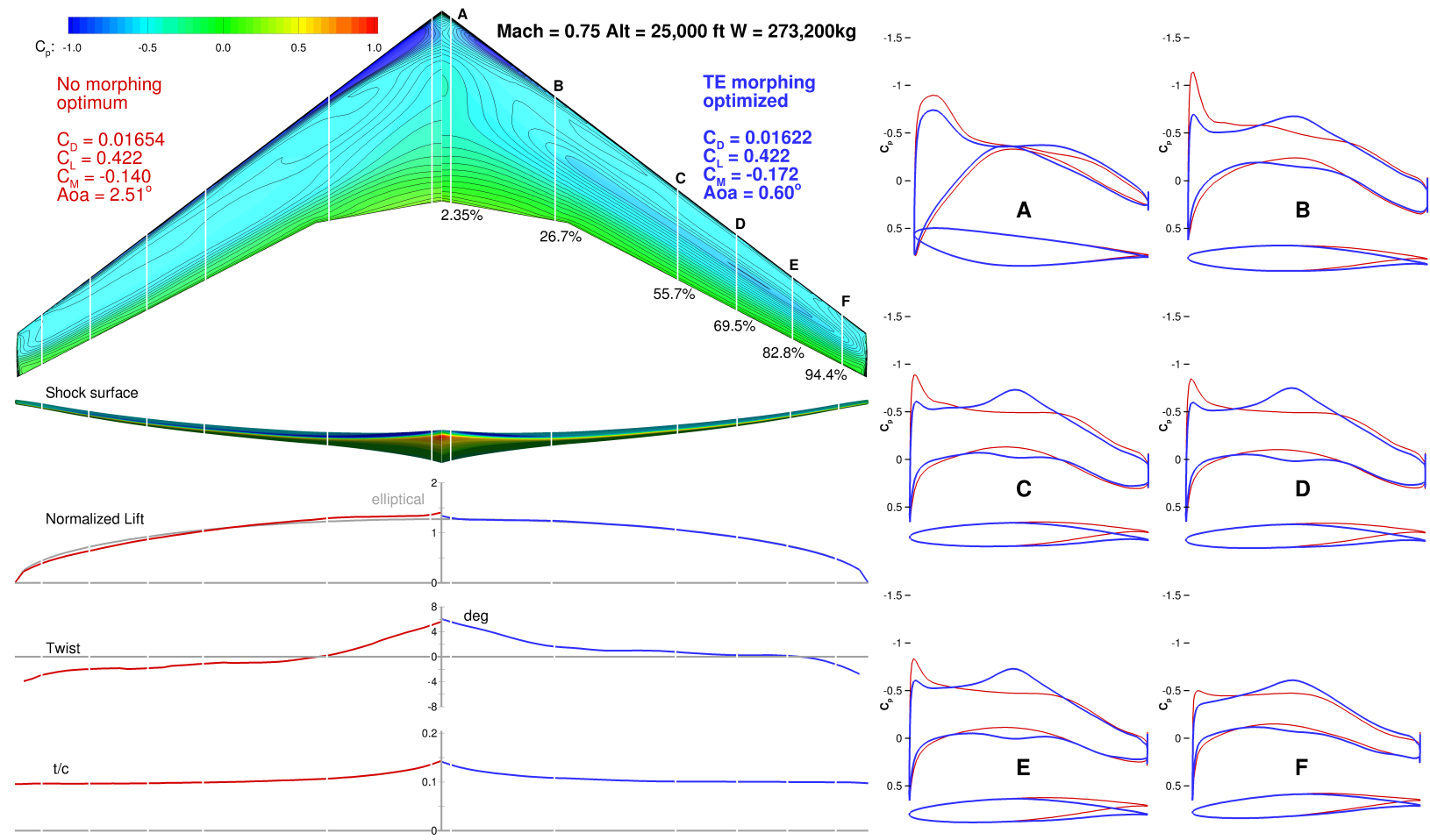

Figure 19. Morphing trailing edge optimization at $M=0.75$, Alt $=25,000 \mathrm{ft}$, and $\mathrm{W}=\mathbf{2 7 3 , 2 0 0} \mathrm{kg}$. 


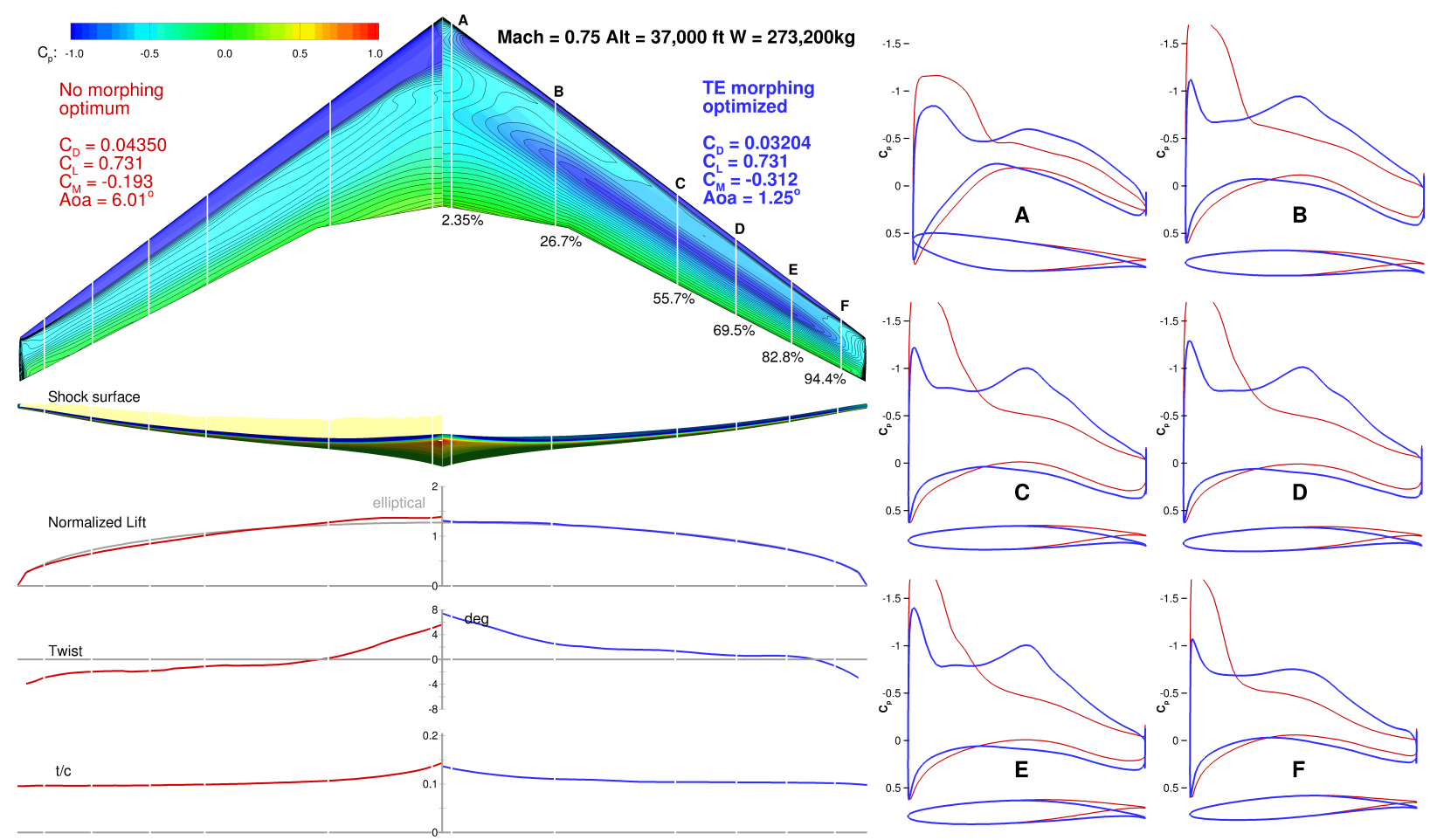

Figure 20. Morphing trailing edge optimization at $M=0.75$, Alt $=37,000 \mathrm{ft}$, and $\mathrm{W}=273,200 \mathrm{~kg}$.
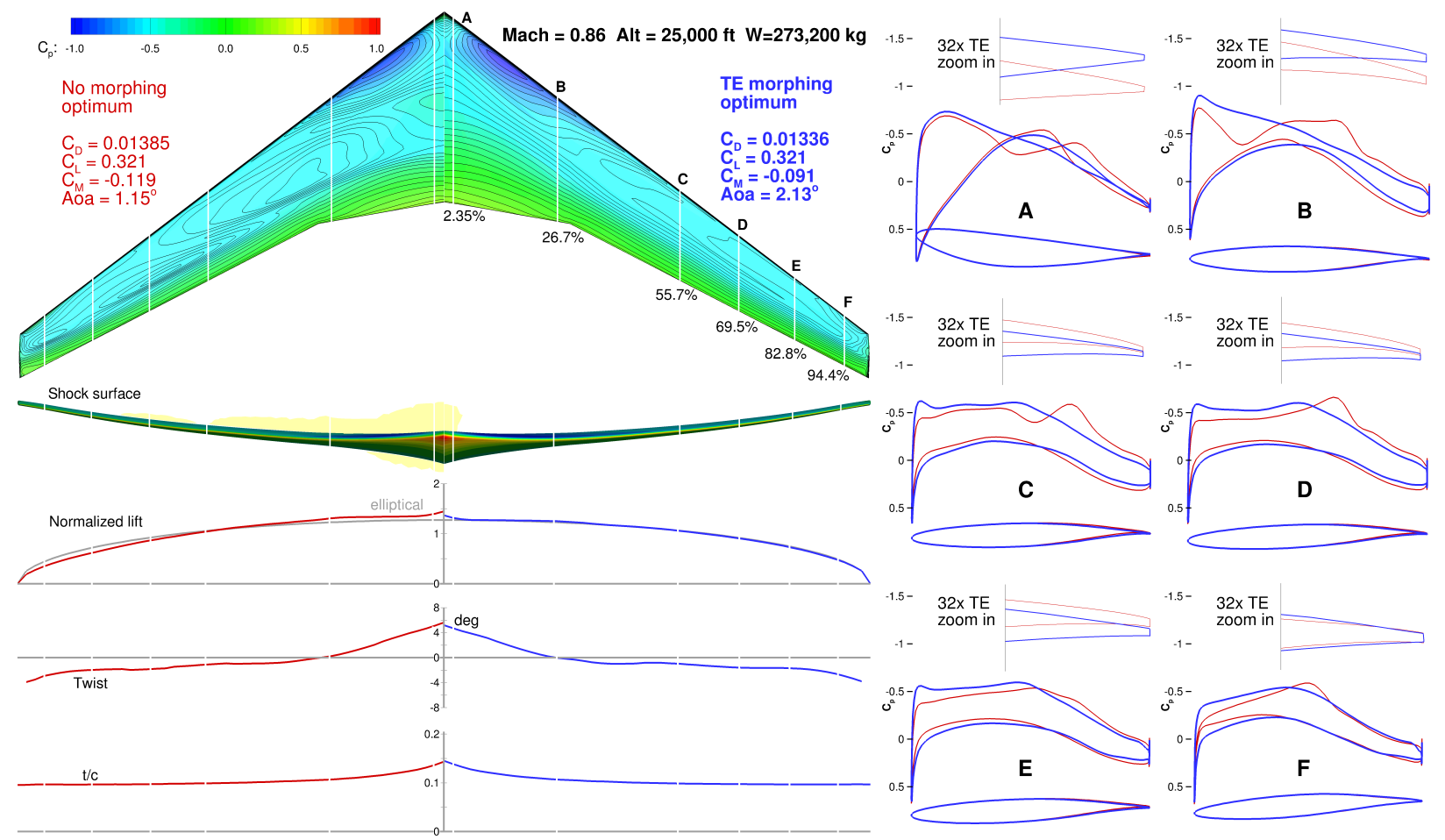

Figure 21. Morphing trailing edge optimization at $\mathrm{M}=\mathbf{0 . 8 6}$, Alt $=25,000 \mathrm{ft}$, and $\mathrm{W}=273,200 \mathrm{~kg}$. 

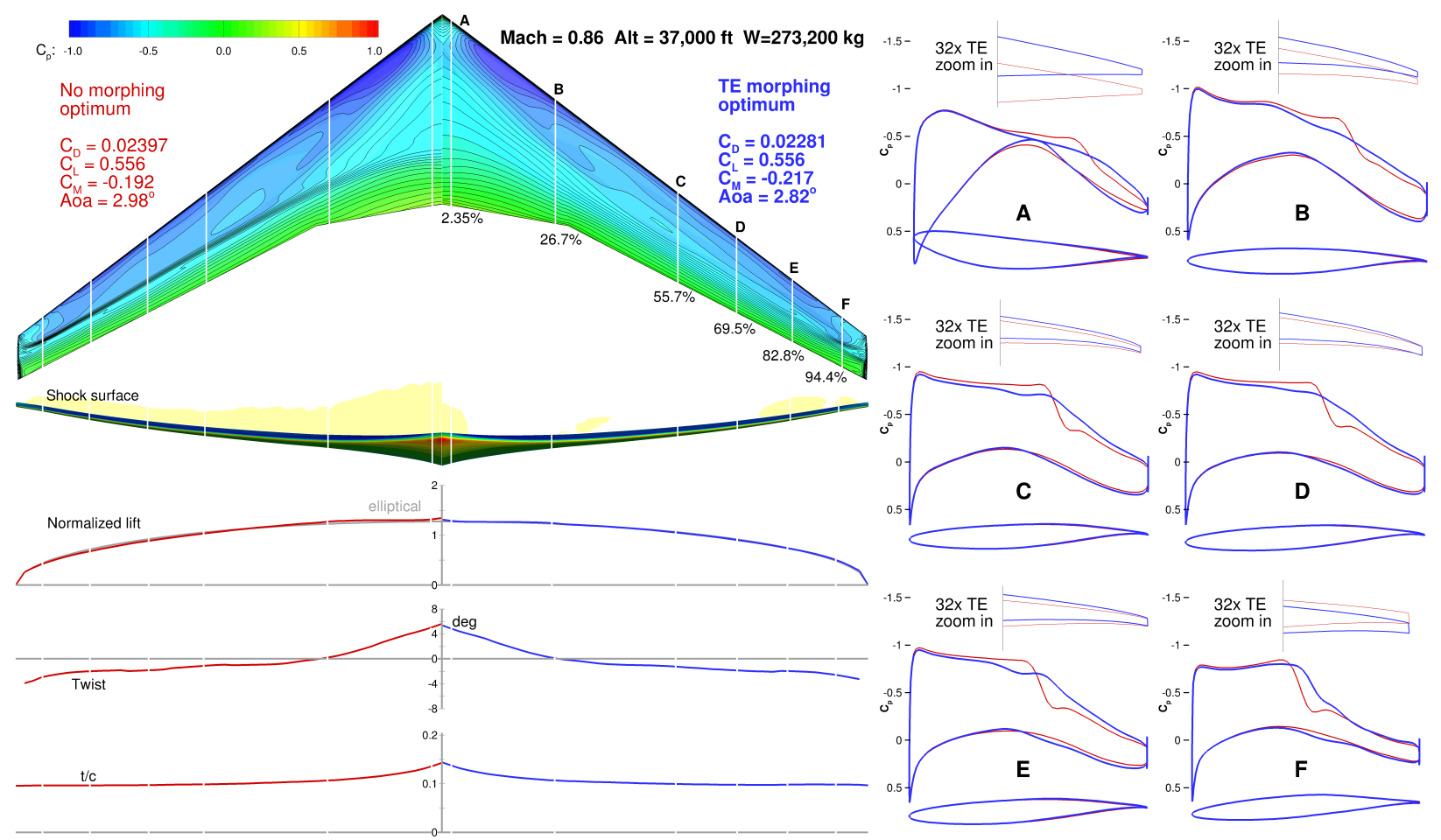

Figure 22. Morphing trailing edge optimization at $M=0.86$, $A l t=37,000 \mathrm{ft}$, and $\mathrm{W}=273,200 \mathrm{~kg}$.
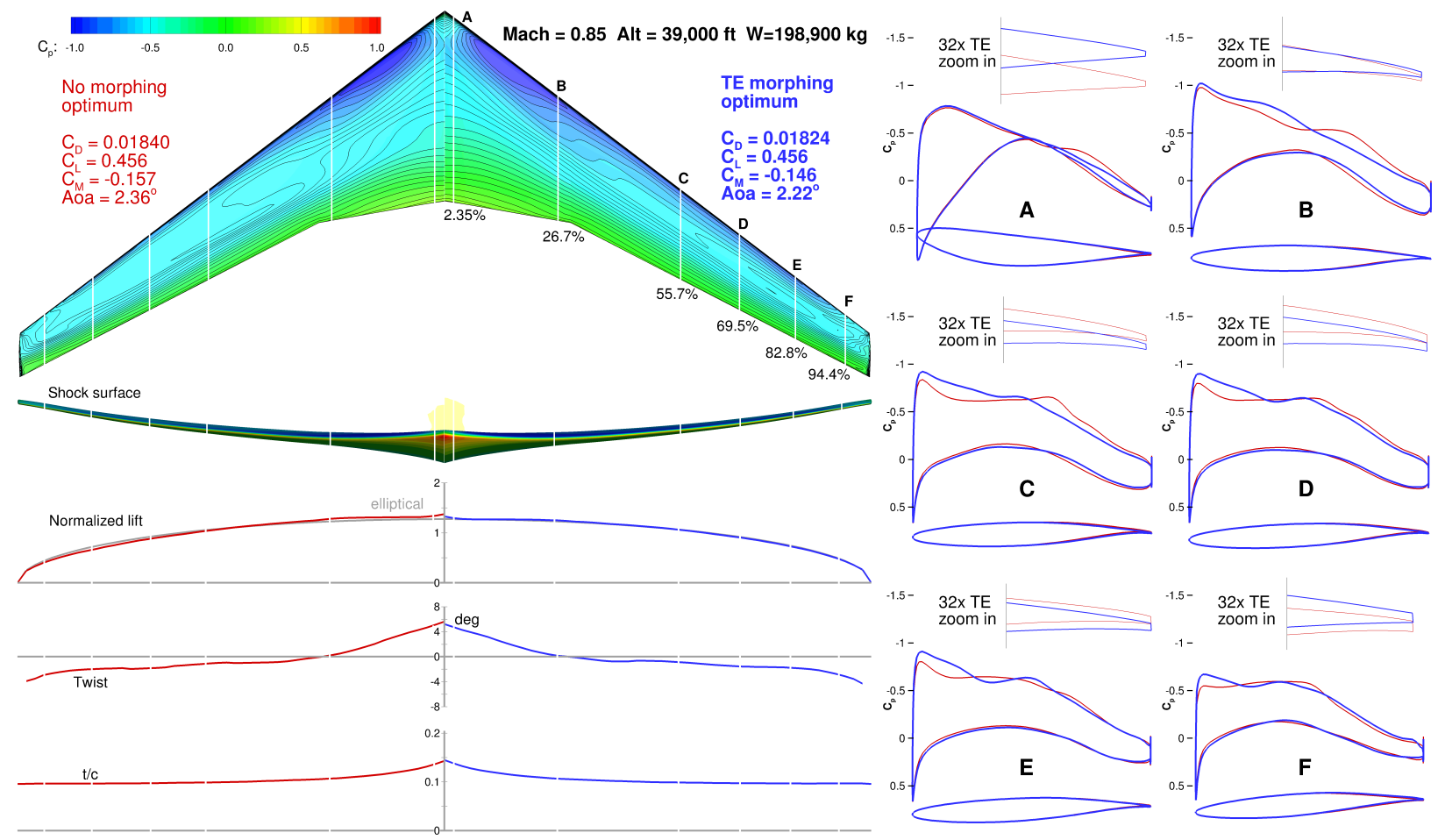

Figure 23. Morphing trailing edge optimization at $M=0.85$, Alt $=39,000 \mathrm{ft}$, and $\mathrm{W}=198,900 \mathrm{~kg}$. 

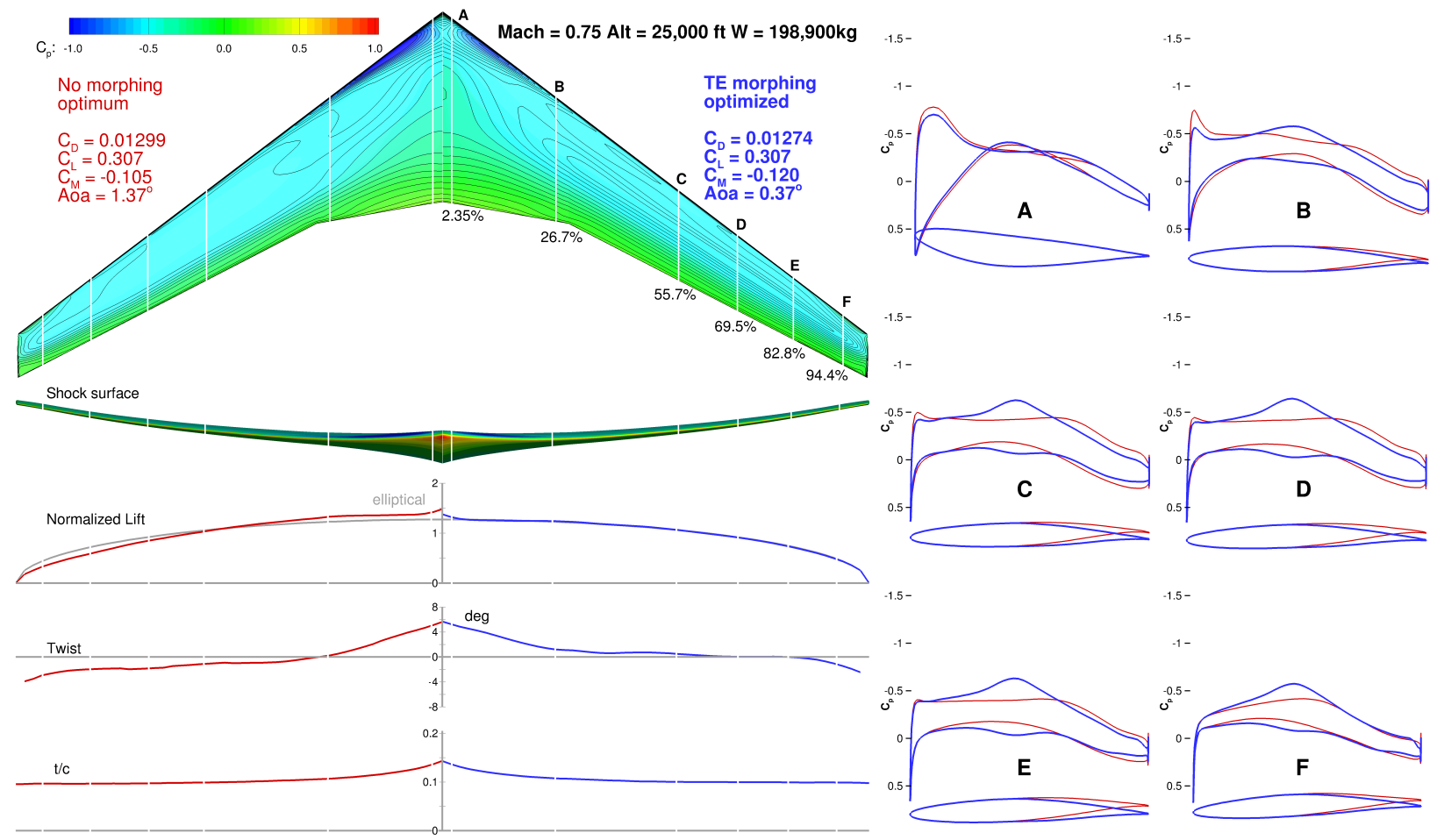

Figure 24. Morphing trailing edge optimization at $M=0.75$, $A l t=25,000 \mathrm{ft}$, and $\mathrm{W}=198,900 \mathrm{~kg}$.
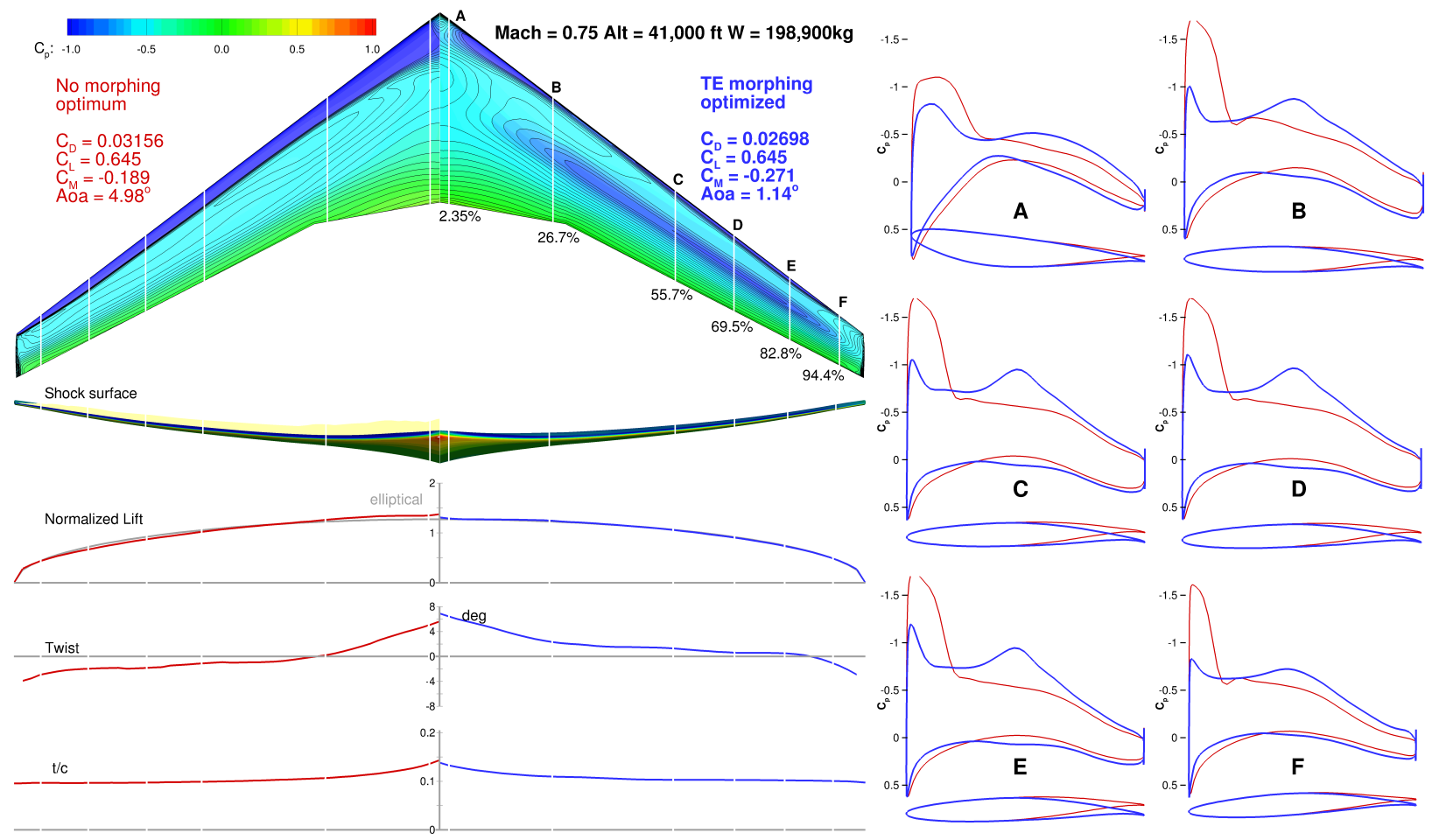

Figure 25. Morphing trailing edge optimization at $\mathrm{M}=\mathbf{0 . 7 5}$, Alt $=41,000 \mathrm{ft}$, and $\mathrm{W}=198,900 \mathrm{~kg}$. 


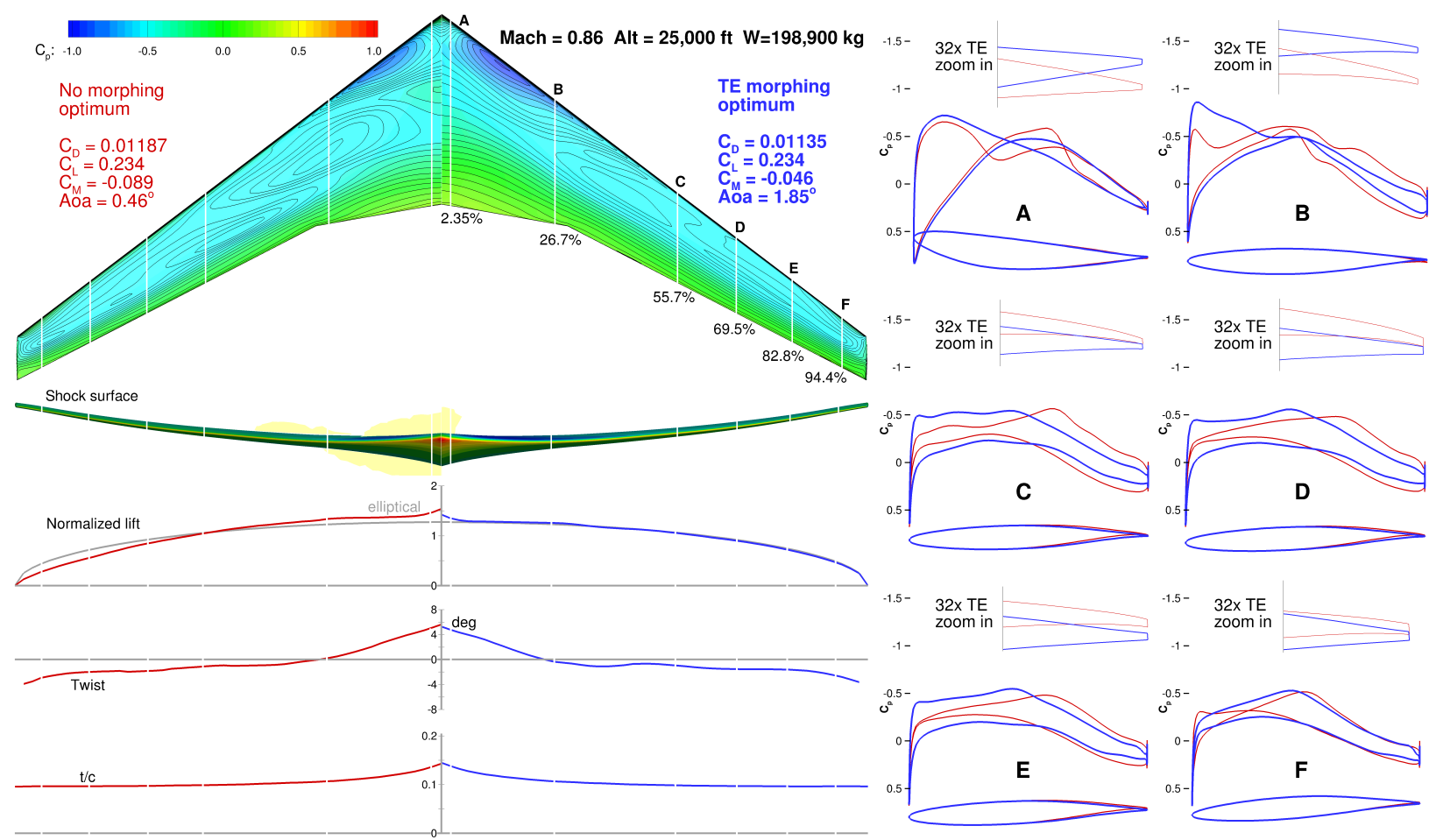

Figure 26. Morphing trailing edge optimization at $M=0.86$, Alt $=25,000 \mathrm{ft}$, and $\mathrm{W}=198,900 \mathrm{~kg}$.

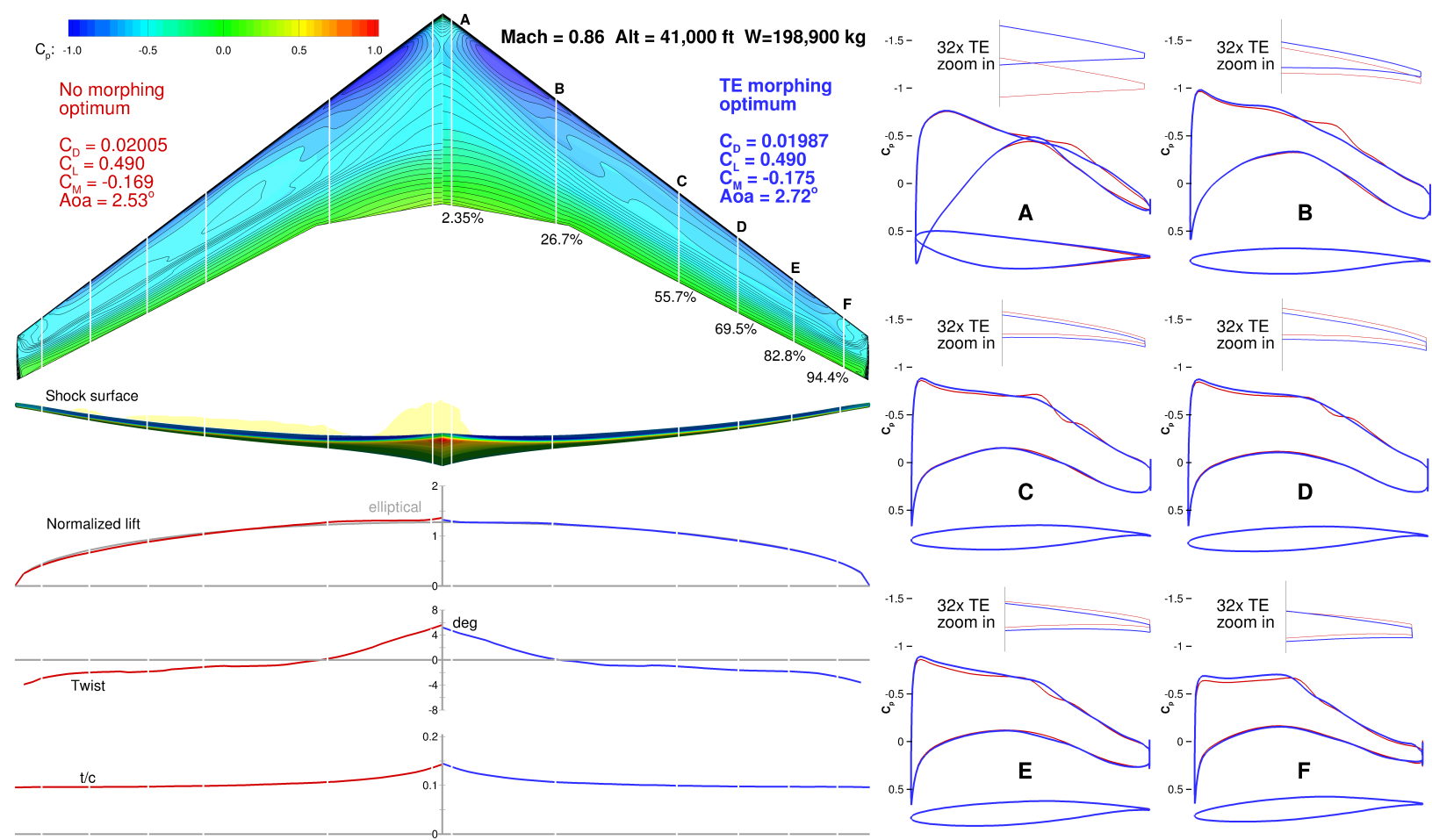

Figure 27. Morphing trailing edge optimization at $M=0.86$, Alt $=41,000 \mathrm{ft}$, and $\mathrm{W}=198,900 \mathrm{~kg}$. 


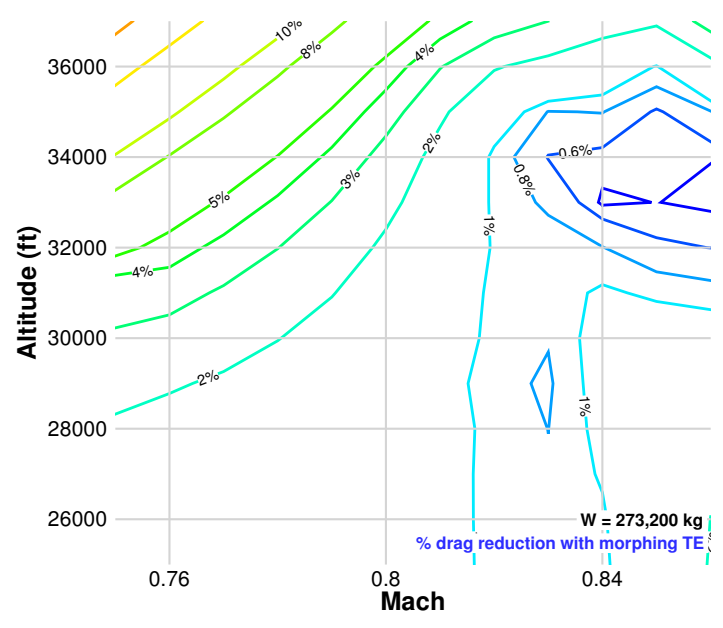

a) Morphing $\mathrm{TE}$

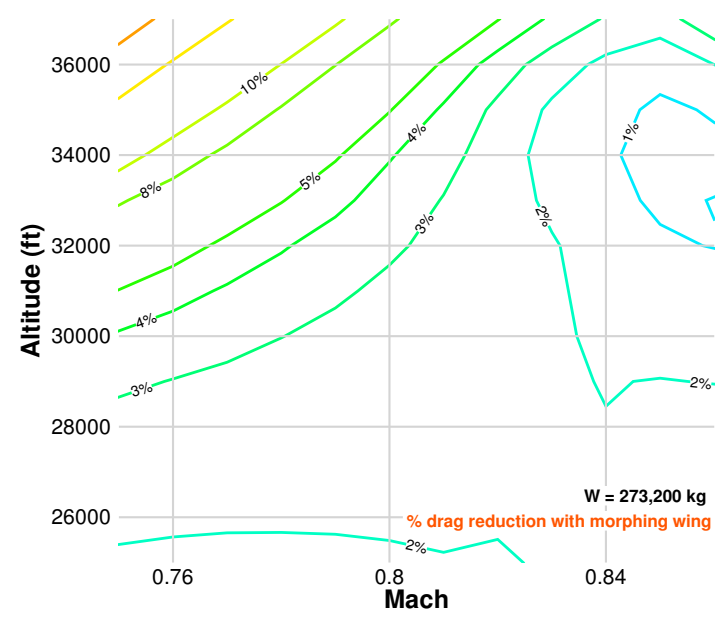

b) Morphing Wing

Figure 28. Drag reduction contour for $\mathrm{W}=\mathbf{2 7 3 , 2 0 0} \mathrm{kg}$.

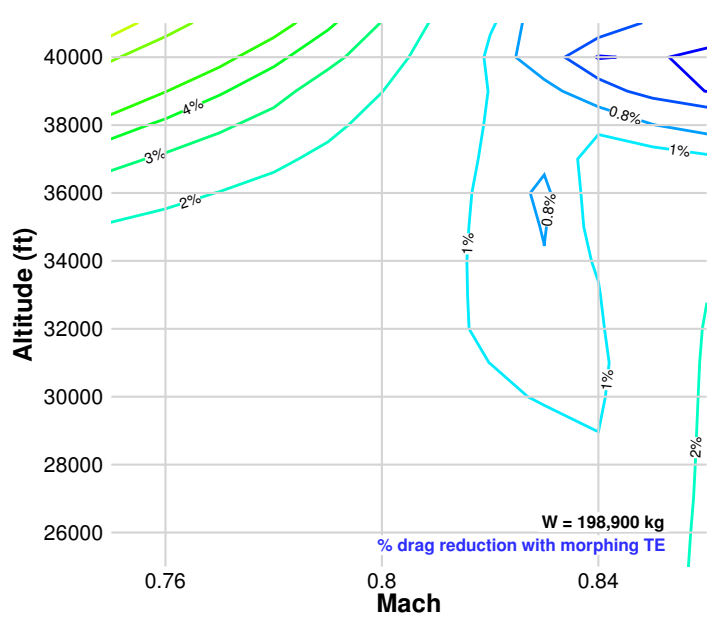

a) Morphing TE

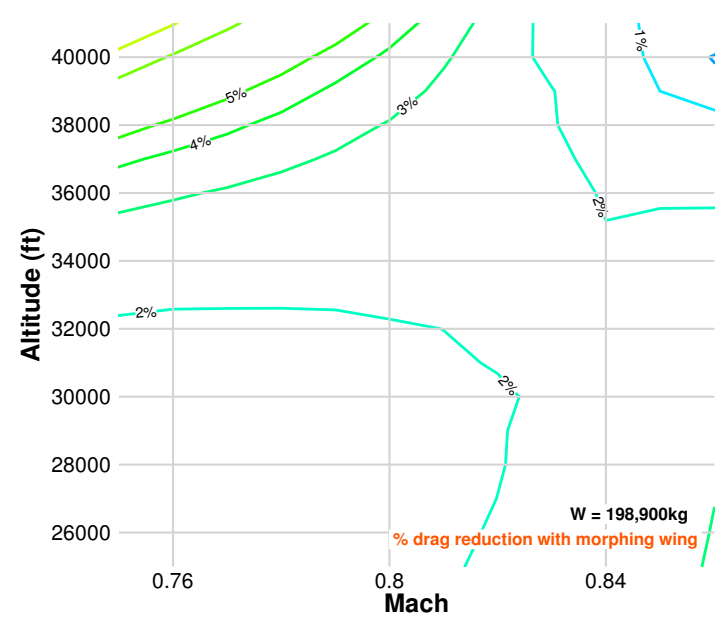

b) Morphing Wing

Figure 29. Drag reduction contour for $W=198,900 \mathrm{~kg}$. 


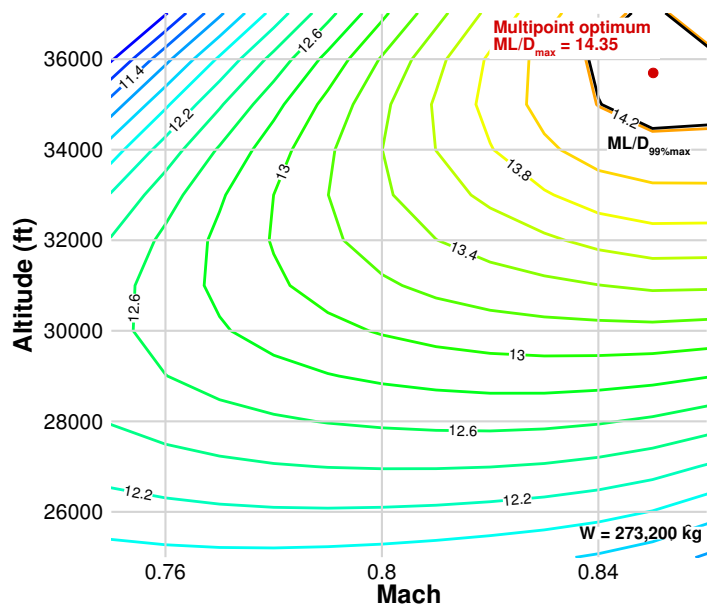

a) Multipoint optimized baseline

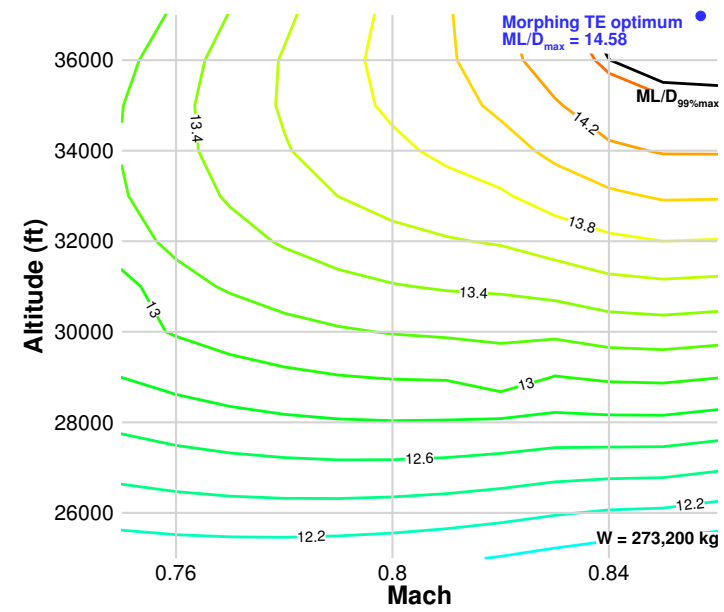

b) Morphing TE

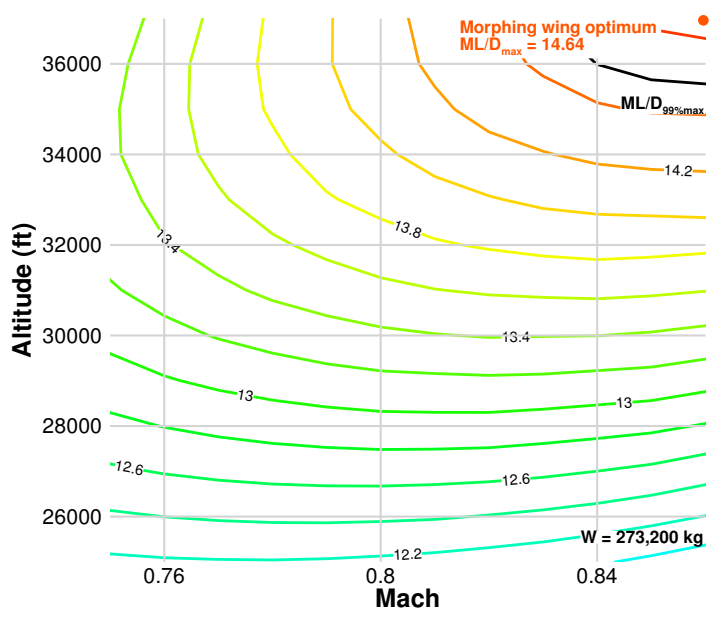

c) Morphing Wing

Figure 30. ML/D contour for $W=273,200 \mathrm{~kg}$. 


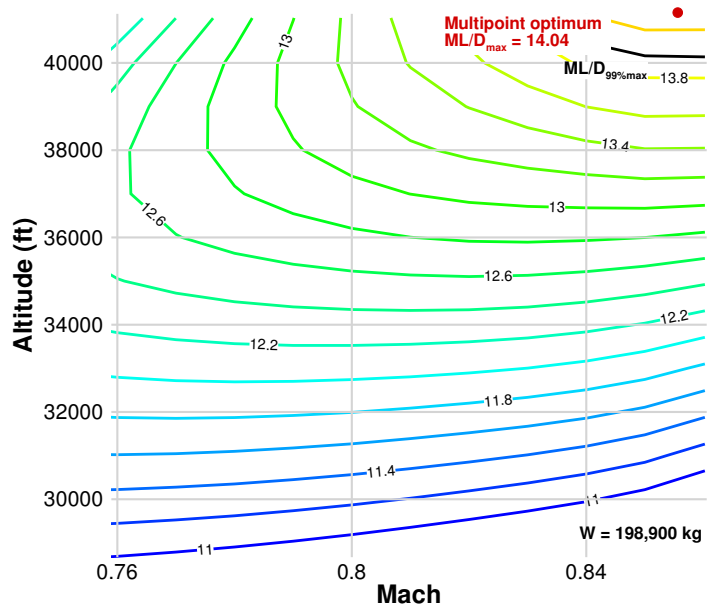

a) Multipoint optimized baseline

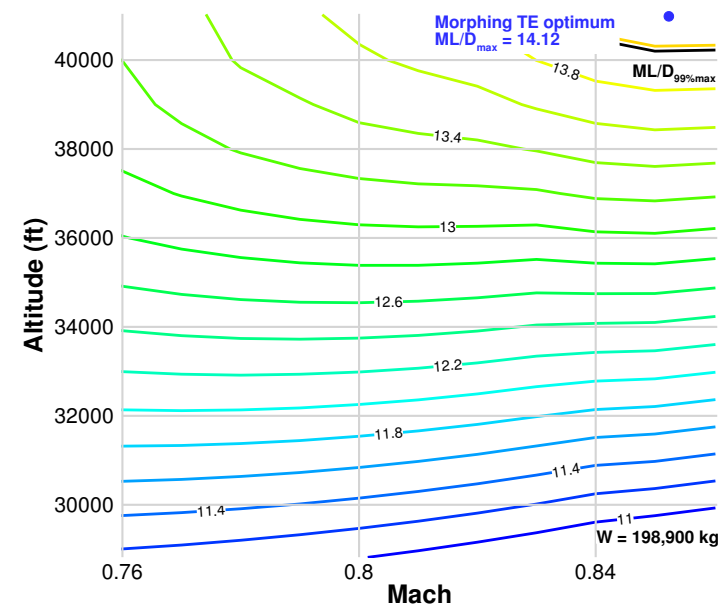

b) Morphing TE

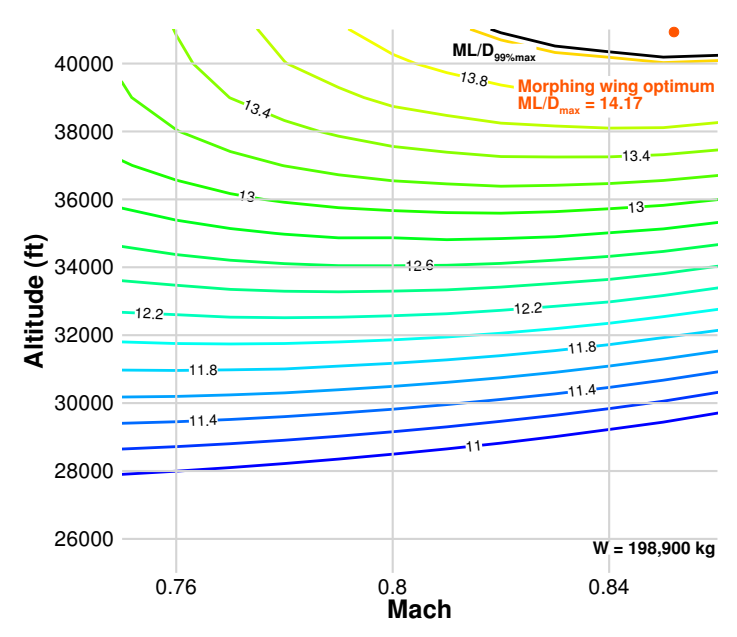

c) Morphing Wing

Figure 31. ML/D contour for $W=198,900 \mathrm{~kg}$. 